1

\title{
Activation of SEDS-PBP cell wall synthases by an essential regulator of bacterial
} division

Patrick J. Lariviere ${ }^{1}$, Christopher R. Mahone ${ }^{1}$, Gustavo Santiago-Collazo ${ }^{2}$, Matthew Howell $^{2}$, Allison K. Daitch ${ }^{1}$, Rilee Zeinert ${ }^{3}$, Peter Chien ${ }^{3}$, Pamela J. B. Brown ${ }^{2}$, Erin D. Goley $^{1 *}$

${ }^{1}$ Department of Biological Chemistry, Johns Hopkins University School of Medicine, Baltimore, MD 21205, USA.

${ }^{2}$ Division of Biological Sciences, University of Missouri, Columbia, MO 65211, USA. ${ }^{3}$ Department of Biochemistry and Molecular Biology, University of Massachusetts Amherst, Amherst, MA 01003, USA.

*For correspondence. E-mail egoley1@jhmi.edu; Tel. (+1) 410-502-4931; Fax (+1) 410955-5759 


\section{Abstract}

22 Bacterial growth and division require insertion of new peptidoglycan (PG) into the

23 existing cell wall by PG synthase enzymes. Emerging evidence suggests that many PG

24 synthases require activation to function, however it is unclear how activation of division-

25 specific PG synthases occurs. The FtsZ cytoskeleton has been implicated as a regulator of

26 PG synthesis during division, but the mechanisms through which it acts are unknown.

27 Here we show that FzlA, an essential regulator of constriction in Caulobacter crescentus,

28 links FtsZ to PG synthesis to promote division. We find that hyperactive mutants of the

29 PG synthases FtsW and FtsI specifically render $f z l A$, but not other division genes, non-

30 essential. However, FzlA is still required to maintain proper constriction rate and

31 efficiency in a hyperactive PG synthase background. Intriguingly, loss of $f z l A$ in the

32 presence of hyperactivated FtsWI causes cells to rotate about the division plane during

33 constriction and sensitizes cells to cell wall-specific antibiotics. We demonstrate that

34 FzlA-dependent signaling to division-specific PG synthesis is conserved in another $\alpha$ -

35 proteobacterium, Agrobacterium tumefaciens. These data establish that FzlA links FtsZ to

36 cell wall remodeling, serving both to activate and spatially orient PG synthesis during

37 division. Overall, our findings support the paradigm that activation of SEDS-PBP PG

38 synthases is a broadly conserved requirement for bacterial morphogenesis. 
43 Bacterial division is driven by the insertion of new cell wall material at midcell in a

44 tightly regulated manner, allowing for determination of cell shape and maintenance of

45 envelope integrity ${ }^{1,2}$. The cell wall is made of peptidoglycan (PG), a meshwork consisting

46 of glycan strands crosslinked by peptide stems ${ }^{3,4}$. PG synthesis requires the coordination

47 of glycan polymerization and peptide crosslinking by either coupled monofunctional

48 glycosyltransferases (GTases) and transpeptidases (TPases), or bifunctional enzymes that

49 contain both activities, with these proteins being more generally referred to as PG

50 synthases ${ }^{1}$.

51 Monofunctional PG synthase pairs have been implicated as the primary synthetic

52 enzymes of the elongation (elongasome) and division (divisome) machineries. A

53 paradigm has been proposed whereby a shape, elongation, division, and sporulation

54 (SEDS) family GTase is functionally coupled to a penicillin binding protein (PBP)

55 TPase, which together facilitate cell wall synthesis ${ }^{5-7}$. Through characterization of the

56 elongation-specific PG synthases RodA and PBP2 in Escherichia coli, it has been

57 postulated that SEDS-PBP enzymes require activation to function ${ }^{5}$. Specifically,

58 mutations in RodA or PBP2 that increase GTase activity in vitro and PG synthesis in

59 cells render other components of the elongasome non-essential, arguing that their normal

60 function is to activate the RodA-PBP2 complex ${ }^{5}$. Intriguingly, analogous mutations in the

61 division-specific SEDS-PBP enzymes, FtsW and FtsI, allow cells to constrict faster than

62 normal $^{8}$, suggesting that these mutations promote formation of an activated PG synthase

63 complex ${ }^{5,9}$. However, it is unclear precisely how SEDS-PBP activation normally occurs

64 during division. 
65 Recent studies have established that the conserved cytoskeletal protein Fts $Z^{10,11}$,

66 which recruits the division machinery to a ring-like structure at midcell ${ }^{12-14}$, is coupled to

67 PG synthesis activation during division. In multiple organisms, the C-terminal linker

68 domain of FtsZ was found to be required for regulating cell wall integrity ${ }^{15-17}$ and shape,

69 as well as PG chemistry ${ }^{16,18}$. Moreover, in E. coli and Bacillus subtilis, FtsZ dynamics

70 were demonstrated to drive PG synthase dynamics in both organisms, as well as division

71 site shape in E. coli and constriction rate in B. subtilis ${ }^{19,20}$. Collectively these data

72 indicate that, at least in some organisms, FtsZ acts as a "dynamic scaffold" or "dynamic

73 activator" of PG synthesis likely impinging on FtsWI. However, the signaling pathway

74 connecting these two endpoints remains unresolved.

75 We previously demonstrated that an essential FtsZ-binding protein, FzlA ${ }^{21}$, is

76 required for division and regulates the rate of constriction in the $\alpha$-proteobacterium

77 Caulobacter crescentus $^{22}$. Mutations in FzlA with diminished affinity for FtsZ were

78 found to have slower constriction rates and altered cell pole shape, indicative of reduced

79 PG synthetic activity during division ${ }^{22}$. We therefore postulated that FzlA facilitates a

80 link between FtsZ and PG synthesis by serving as an upstream activator of PG synthases

81 and, here, set out to test this hypothesis.

82 


\section{Results}

\section{$84 f \boldsymbol{f} \boldsymbol{l} \boldsymbol{A}$ lies upstream of $\boldsymbol{f t s} \boldsymbol{W}$ in a PG synthesis pathway}

85 We reasoned that if FzlA impacts constriction through PG synthases, it likely acts on the

86 division-specific SEDS family GTase FtsW and/or the monofunctional PBP TPase FtsI.

87 To assess if FzlA activates FtsWI, we leveraged fast-constricting strains containing

88 hyperactive mutant variants of FtsI and/or FtsW termed $f t s W^{* *} I^{* 8,9}$ and $f t s W^{* 9} . f t s W^{* *} I^{*}$

89 bears the mutations F145L and A246T in FtsW and I45V in FtsI, whereas $f t s W^{*}$ contains

90 only the FtsW A246T mutation ${ }^{9}$. These mutations are thought to stabilize an activated

91 form of the FtsWI complex ${ }^{5,9}$, leading to increased rates of cell constriction ${ }^{8}$ via

92 unrestrained PG synthesis.

93 If $f z l A$ lies upstream of $f t s W I$ in a PG synthesis pathway, then the hyperactive

94 variants $f t s W^{* *} I^{*}$ and/or $f t s W^{*}$ may bypass the essentiality of $f z l A$. Accordingly, we

95 found that $f z l A$ could be readily deleted in either the $f t s W^{* *} I^{*}$ or $f t s W^{*}$ strain

96 backgrounds (Fig. 1A-B, Fig. S1A). This is a particularly striking finding given that

97 depletion of FzlA in a WT background completely inhibits division and induces cell

98 filamentation and death ${ }^{21}$. Interestingly, a number of $f t s W^{* *} I^{*} / f t s W^{*} \Delta f z l A$ cells appeared

99 to be "S"-shaped with the direction of curvature in future daughter cells facing opposite

100 directions, as opposed to the characteristic "C"-shape of pre-divisional WT and $f t s W^{* *} I^{*}$

101 Caulobacter cells (Fig. 1A, asterisk, discussed further below). Assessment of fitness

102 revealed that strains lacking $f z l A$ displayed a slight reduction in viability by spot dilution,

103 compared to the corresponding hyperactive PG synthase mutant strains (Fig. 1C),

104 whereas growth rate was unaffected (Fig. 1D). In addition, $f t s W^{* *} I^{*} / f t s W^{*} \Delta f z l A$ cells

105 displayed an increase in length (Fig. 1E), indicative of a residual division defect. Because 
$106 f t s W^{*} \Delta f z l A$ cells are longer than $f t s W^{* *} I^{*} \Delta f z l A$ cells, we conclude that $f t s W^{* *} I^{*}$

107 suppresses loss of $f z l A$ better than the single mutant.

108 We also observed that $f t s W^{* *} I^{*}$ suppresses length, width, and fitness defects

109 associated with slowly constricting $f z l A$ point mutants $f z l A^{N H 2}$ and $f z l A^{N H 3}$ (Fig. S2, Fig.

110 S1B), further indicating that hyperactivated $f t s W I$ are dominant to, and likely downstream

111 of $f z l A$. To determine the contribution of the FtsZ-FzlA interaction to activation of FtsWI,

112 we assessed cell morphology, fitness, and cell length of $f t s W^{* *} I^{*}$ strains containing FzlA

113 mutants with decreasing affinity for FtsZ ${ }^{22}$ (Fig. S3, Fig. S1C; FzlA $>$ FzlA ${ }^{\mathrm{NH} 2}=$

$114 \mathrm{FzlA}^{\mathrm{NH} 3}>\mathrm{FzlA}^{\mathrm{NH} 1} ; \mathrm{FzlA}^{\mathrm{NB} 2}, \mathrm{FzlA}^{\mathrm{NB} 1}=$ no binding). We found that decreased affinity of

115 FzlA towards FtsZ correlated with an increase in cell length (Fig. S3E), indicating that

116 high-affinity binding to FtsZ is required for FzlA to signal to FtsWI.

118 FzlA plays a specific and unique role in activating FtsWI

119 To assess the specificity of the $f z l A-f t s W I$ genetic interaction and potentially

120 identify additional components of this pathway, we performed comparative transposon

121 sequencing (Tn-Seq) on WT and $f t s W^{* *} I^{*}$ strains. Surprisingly, $f z l A$ was the only

122 essential gene to become non-essential in the $f t s W^{* *} I^{*}$ background, with few insertions

123 in WT but plentiful insertions in $f t s W^{* *} I^{*}$ cells (Fig. 1F,G, Supplementary Table 1).

124 All other known essential division genes, e.g. ftsZ (Fig. 1G), had few transposon

125 insertions in either background. These data indicate that $f z l A$ is specific and unique in its

126 essential role upstream of $f t s W I$. We suspect that other essential division proteins

127 participate in this pathway as well, but that they play additional essential functions in

128 divisome assembly or activity. 


\section{FzlA contributes to efficient division in a hyperactive PG synthase background}

130 Given that cells lacking $f z l A$ in the hyperactive PG synthase backgrounds were elongated,

131 we assessed constriction rate and division efficiency in these strains in more detail.

132 Specifically, we performed time-lapse microscopy on $f t s W^{* *} I^{*}$ and $f t s W^{*}$ cells $\pm f z l A$ and

133 tracked division in cells using Microbe $^{22,23}$ (Fig. 2A, Supplementary Video 1).

134 Consistent with previous findings ${ }^{8}, f t s W^{* *} I^{*}$ and $f t s W^{*}$ cells constrict more quickly than

135 WT (Fig. 2B). Intriguingly, the hyperactive PG synthase strains lacking $f z l A$ constricted

136 significantly more slowly than the corresponding strain with $f z l A$ present, with

137 constriction rates cut nearly in half (Fig. 2B). This suggests that hyperactivated FtsWI are

138 not sufficient for efficient division and underscores the importance of FzlA in dictating

139 constriction rate. As with cell length and fitness, $f t s W^{* *} I^{*}$ acted as a better suppressor to

$140 f z l A$ deletion, allowing for a faster constriction rate than $\operatorname{did} f t s W^{*}$ (Fig. 2B).

141 To ensure that changes in constriction rate were not due to global differences in

142 PG synthesis, we determined elongation rates across strains (Fig. 2C), which enabled

143 calculation of the ratio of constriction to elongation rate (Fig. 2D). We saw the same

144 trend as for constriction rate itself, with $f t s I^{* *}$ and $f t s W^{*}$ mutant strains having higher

145 ratios of constriction to elongation and loss of $f z l A$ giving lower ratios (Fig. 2D).

146 Interestingly, elongation rate was inversely correlated with constriction rate in all mutant

147 strains (Fig. 2C, Fig. 2D), perhaps reflecting competition between the elongasome and

148 divisome for PG precursor substrate ${ }^{24}$. Altogether, these data support the conclusion that

149 alterations to the $f t s Z-f z l A-f t s W I$ pathway specifically affect constriction, with FzlA

150 increasing the constriction rate in both WT and hyperactive PG synthase mutant

151 backgrounds. 
While tracking division in $f t s W^{* *} I^{*} \Delta f z l A$ and $f t s W^{*} \Delta f z l A$ cells to measure

153 constriction, we noticed that some cells initiated constriction at one location, then aborted

154 division at that location before successfully dividing at a second (or third or fourth) site

155 (Fig. 2E, Supplementary Video 2). We quantified the frequency of such constriction

156 failure events and found that $16.6-19.5 \%$ of the hyperactive PG synthase cells lacking

$157 f z l A$ aborted division at one site before successfully dividing at another, compared to a 0-

$158 \quad 0.3 \%$ failure rate for WT or hyperactive PG synthase cells with $f z l A$ present (Fig. 2F).

159 These data further demonstrate that $f t W^{*} I^{*}$ are not sufficient for efficient division, and

160 that $f z l A$ is required to ensure division processivity and efficiency.

$162 f \boldsymbol{f} \boldsymbol{l} \boldsymbol{A}$ is required for maintenance of proper cell shape

163 As mentioned earlier, deletion of $f z l A$ in the hyperactive PG synthase backgrounds

164 impacted global cell morphology, with many pre-divisional cells appearing "S-shaped".

165 In order to more carefully assess this phenotype, we imaged cells by scanning electron

166 microscopy (SEM). We saw a relatively high frequency of S-shaped $f t s W^{* *} I^{*} \Delta f z l A$ cells,

167 whereas most WT or $f t s W^{* *} I^{*}$ cells displayed the typical "C-shaped" morphology

168 characteristic of Caulobacter (Fig. 3A). We quantified the frequency of S-shaped cells in

169 a population of dividing cells by phase contrast microscopy to assess penetrance of this

170 morphological phenotype. We extracted outlines of individual cells and performed

171 principal component analysis using Celltool to isolate variance in cell shape to features

172 referred to as shape modes ${ }^{22,25}$. Shape mode 3 captured the variation due to degree of S-

173 versus $\mathrm{C}$-shape and we set a cutoff such that cells with a standard deviation $|\mathrm{sd}|>1$ from

174 the mean for this shape mode are considered S-shaped (Fig. 3B,C). Means and medians 
175 were similar for degree of S-shape across populations, with no significant difference for

176 means, and a statistically significant but numerically small difference for medians.

177 However, there was an obvious and significant difference in variance in degree of S-

178 shape across populations (Fig. 3B), corresponding with a large difference in the number

179 of cells found to be S-shaped in different strains. Over a quarter (26.9\%) of dividing

$180 f t s W^{* *} I^{*} \Delta f z l A$ cells displayed an S-shaped morphology, compared to $2.4 \%$ of WT and

$181 \quad 1.1 \%$ of $f t s W^{* *} I^{*}$ cells that are S-shaped (Fig. 3D).

182 To shed light on the origin of S-shape, we next asked at what point during growth

183 do $f t s W^{* *} I^{*} \Delta f z l A$ cells begin to adopt this morphology. Using time-lapse microscopy,

184 we observed that $f t s W^{* *} I^{*} \Delta f z l A$ cells were C-shaped at the beginning of the cell cycle

185 and began to twist or rotate about the division plane after constriction initiated. S-shape

186 only became apparent in the latter part of constriction, when daughters had rotated $\sim 180^{\circ}$

187 relative to each other (Fig. 3E, Supplementary Video 3). This finding suggests that the

$188 f$ flA-ftsWI pathway determines geometry of PG insertion at the site of division in a

189 manner that influences global cell morphology, normally constraining cells in their

190 characteristic C-shape as constriction progresses. These results also indicate that our

191 quantification method for S-shape likely underestimates the number of twisted $f t s W^{* *} I^{*}$

$192 \Delta f z l A$ cells, since S-shape is not obvious by phase contrast until the end of constriction,

193 and we quantified cell shape at all stages of the constriction process.

194 Changes in division site shape and formation of S-shaped cells have been

195 previously linked to aberrant localization of FtsZ and the elongation factor, MreB,

196 respectively ${ }^{19,26}$. To determine if cell twisting might be facilitated by mislocalized

197 division or elongation machineries, we visualized FtsZ and MreB localization in fts $W^{* *} I^{*}$ 
$198 \Delta f z l A$ cells using inducible fluorescent fusions of these proteins. However, mNG-FtsZ

199 and Venus-MreB localization in $f t s W^{* *} I^{*} \Delta f z l A$ cells was comparable to $f t s W^{* *} I^{*}$ cells

200 (Fig. S4, S5). Additionally, we visualized the localization of PG synthesis using the

201 fluorescent D-amino acid $\mathrm{HADA}^{27}$, in order to assess whether cell twisting might be

202 induced by mislocalized PG synthesis in spite of properly localized FtsZ and MreB.

203 However, we did not detect any gross changes in HADA localization (Fig. S6). Together,

204 these findings suggest that cell twisting is likely induced by a finer scale alteration of PG

205 synthesis at the division site due to disruption of the fzlA-ftsWI pathway.

206 To determine whether global shape regulation depends on the FtsZ-FzlA

207 interaction, we assessed S-shaped cell frequency in strains containing mutants of FzlA

208 displaying decreasing affinities towards FtsZ. We observed that affinity of the mutant

209 FzlA for FtsZ was inversely correlated with the frequency of S-shaped cells (Fig. S7),

210 verifying that the FtsZ-FzlA interaction is important for maintaining proper morphology.

212 The $f t s Z$ - $f$ zlA-ftsWI pathway contributes to resistance to PBP-targeting antibiotics

213 Because FzlA is important for regulation of PG synthesis in the context of determining

214 constriction rate and cell shape, we hypothesized that it might also contribute to

215 resistance to cell wall-targeting antibiotics. To test this, we challenged cells with

216 antibiotics targeting PG synthetic processes and assessed resulting cell fitness. fts $W^{* *} I^{*}$,

217 as has been previously shown ${ }^{9}$, displayed sensitivity to cephalexin (Fig. 4A), which

218 inhibits FtsI and other penicillin-binding proteins in Caulobacter ${ }^{28,29}$. Interestingly,

219 deletion of $f z l A$ in the $f t s W^{* *} I^{*}$ background exacerbated sensitivity to cephalexin (Fig.

220 4A). We found a similar trend upon treatment with mecillinam, which targets the 
221 elongation-specific PG synthase PBP2 $2^{30,31}$, whereby the minimum inhibitory

222 concentration (MIC) for $f t s W^{* *} I^{*}$ cells was decreased compared to WT, with deletion of

$223 f z l A$ further lowering the MIC (Fig. 4B). We also treated cells with the $\beta$-lactam

224 ampicillin ${ }^{32}$ and with the cell wall targeting antibiotics vancomycin, which blocks

225 transpeptidation by a distinct mechanism from $\beta$-lactams ${ }^{33}$, and fosfomycin, which

226 inhibits cell wall synthesis by blocking PG precursor availability ${ }^{34}$ (Fig. S8). Neither

227 hyperactivation of $f t$ ts $I$ nor loss of $f z l A$ yielded a change in MIC in the presence of any

228 of these antibiotics (Fig. S8). $f z l A$ therefore supports robust cell wall synthesis in the

229 presence of certain PG-targeting drugs, perhaps by compensating for inactivation of

230 specific PBPs. To determine if the interaction between FtsZ and FzlA is important for

231 maintaining cell wall integrity, we assessed sensitivity to cephalexin using the panel of

$232 f z l A$ mutants which display varying affinities towards FtsZ (Fig. S9). We found that

233 mutants with decreased FzlA affinity towards FtsZ in fact became more sensitive to

234 cephalexin (Fig. S9), demonstrating that the entire $f t s Z-f z l A-f t s W I$ pathway is required for

235 promoting cell wall integrity during antibiotic treatment.

236 Since $f t s W^{* *} I^{*}$ cells are more sensitive to perturbation of other PG synthetic

237 activities even when $f z l A$ is present, we asked if any normally non-essential division

238 genes become more important for fitness in an $f t s W^{* *} I^{*}$ background, as they might help

239 bolster resistance to assaults on PG synthesis. Examination of the $f t s W^{* *} I^{*} \mathrm{Tn}-\mathrm{Seq}$ data

240 indicated that $\operatorname{pbp} X$ (encoding a bifunctional PG synthase that localizes to midcell) ${ }^{35,36}$,

241 and to a lesser extent $f t s X$ (encoding a cell separation factor) ${ }^{37}$ and $\operatorname{dip} M$ (encoding an

242 envelope maintenance/cell separation factor $)^{37-40}$, had fewer transposon insertions in an

$243 f t s W^{* *} I^{*}$ background than in WT (Fig. 1F, S10). Because $f t s W^{* *} I^{*}$ cells have 
244 misregulated division site PG synthase activity, we suspect that FtsEX, DipM, and PbpX

245 become important for ensuring robust PG synthesis during constriction and, later,

246 efficient cell separation. Surprisingly, the normally non-essential nhaA locus, coding for a

247 putative sodium-proton antiporter ${ }^{41}$, was also predicted by Tn-Seq to become essential in

$248 f t s W^{* *} I^{*}$ cells (Fig. 1F, Fig. S10B). Disruption of $n h a A$ in the presence of sucrose has

249 been shown to arrest division ${ }^{41}$, suggesting nhaA may be important for division under

250 certain conditions. It is unclear why it also becomes important upon PG synthesis mis-

251 regulation, but its role in osmoregulation may contribute to its apparent synthetic lethality

252 with $f t s W^{* *} I^{*}$.

\section{The $\boldsymbol{f} z \boldsymbol{l} \boldsymbol{A}$ - $f$ ts $\boldsymbol{W}$ genetic interaction is conserved in diverse $\alpha$-proteobacteria}

255 FzlA homologs are encoded in nearly all sequenced $\alpha$-proteobacterial genomes, but not

256 outside this group. To assess the conservation of FzlA's role in regulating PG synthesis,

257 we sought to characterize the genetic interaction between $f z l A$ and PG synthases in

258 another $\alpha$-proteobacterium, Agrobacterium tumefaciens. A. tumefaciens and Caulobacter

259 display disparate growth patterns driven by distinct machineries during elongation, with

260 A. tumefaciens exhibiting polar elongation and Caulobacter elongating primarily at

261 midcell and through dispersed growth ${ }^{27,42-44}$. However, the components of the division

262 machinery, including FtsZ, FzlA, and FtsWI, are largely conserved. To test if the genetic

263 interaction between $f z l A$ and $f t s W$ is conserved, we made an IPTG-dependent FzlA

264 depletion construct in a WT background or in a background with a single hyperactivating

265 mutation in $A$. tumefaciens fts $W$ (F137L, the equivalent of Caulobacter FtsW F145L) at

266 the $f t s W$ locus. Depletion of FzlA in a WT background resulted in reduced viability, 
267 division arrest, and ectopic pole formation at midcell (Fig. 5, Fig. S11), reminiscent of

268 FtsW depletion in A. tumefaciens ${ }^{18}$. Importantly, we found that the decrease in viability

269 and morphology defects associated with depletion of FzlA were rescued by fts W F $137 \mathrm{~L}$

270 (Fig. 5, Fig. S11). These data indicate that FzlA's essential role in regulating division-

271 specific PG synthesis is conserved in another $\alpha$-proteobacterium and further highlight the

272 importance of FzlA as a key regulator of constriction and cell morphology.

273 


\section{Discussion}

275 Here we have described a conserved PG synthesis activation pathway in which FtsZ and

276 FzlA signal through FtsWI to regulate wall synthesis during division in $\alpha$-proteobacteria

277 (Fig. 6, left panel). Specifically, the FtsZ-FzlA-FtsWI pathway determines geometry of

278 cell wall insertion at the site of division, sets the constriction rate, and promotes cell wall

279 integrity (Fig. 6, left panel). FtsW**I* can still receive input from FzlA which, in

280 combination with their intrinsic hyperactivity, leads to shorter, faster-constricting cells

281 with sensitivity to cell wall antibiotics (Fig. 6, middle panel). In the absence of $f z l A$,

$282 f t s W^{* *} I^{*}$ cells lose critical regulation of PG synthesis, leading to twisting during division,

283 slower constriction, and increased sensitivity to cell wall antibiotics. We establish FzlA

284 as a key intermediary in signaling from FtsZ to FtsWI and demonstrate that this division-

285 specific SEDS-PBP pair require activation for normal division. Notably, our observations

286 indicate that FtsWI activity is regulated in multiple ways, likely including input into their

287 catalytic rates and modulation of the fine-scale geometry by which they insert new PG for

288 constriction.

289 Our findings provide the foundation for further mechanistic investigation into this

290 pathway and raise a number of questions. For one, the nature and timing of the activation

291 signal(s) are still unknown: is there a signal always emanating from FtsZ-FzlA that

292 induces constriction as soon as FtsW arrives, or is constriction triggered by a discrete

293 cellular event, such as clearance of the chromosomal termini or the arrival of a sparkplug

294 factor that jumpstarts FtsWI activity? Additionally, it is unclear why hyperactivation of

$295 f t s W I$ together or $f t s W$ alone can rescue loss of $f z l A$ - are TPase and GTase activities

296 impacted similarly, or does one predominate in regulating constriction rate? A recent 
297 study demonstrated that FtsW is activated by $\mathrm{FtsI}^{7}$, so it is possible that the $\mathrm{ftsI}^{*}$ mutation

298 described here in fact hyperactivates FtsW. Finally, we have no evidence that FzlA and

299 FtsWI directly interact, and suspect that other intermediary factor(s) transduce the

300 activation signal from FzlA to FtsWI.

301 Our model advances the idea that regulation of SEDS-PBP pairs for growth and

302 division is conserved at numerous levels. The finding that FzlA governs division-specific

303 PG synthesis in both Caulobacter and A. tumefaciens argues that $\alpha$-proteobacteria use

304 FzlA as a conserved and dedicated FtsWI activator. FzlA is absent outside of this clade,

305 however, so we propose that other divisome components serve as FtsWI activators in

306 other organisms. More broadly, our findings expand the paradigm for PG synthesis by

307 SEDS-PBP PG synthase pairs in bacteria and provide evidence that the requirement for

308 PG synthase activation is conserved. Elongation is facilitated by the coordination of the

309 SEDS family GTase RodA and the monofunctional TPase PBP2, orthologs to FtsW and

310 FtsI, respectively 5 . The proposed model for elongation activation, as described for E. coli,

311 holds that these PG synthases are activated by another protein, MreC, forming an

312 activated complex that in turn regulates assembly and directional motion of the

313 polymerizing scaffold $\mathrm{MreB}^{5}$. In this system, hyperactivating mutations in RodA or PBP2

314 allow for bypass of the activator, $\mathrm{MreC}$, similar to our finding that $\mathrm{FtsW}^{* *} \mathrm{I}^{*}$ can bypass

315 the activator FzlA. Our data provide experimental support for the proposal that the

316 requirement for activation of the SEDS-PBP pair of PG synthases is generally conserved

317 for elongation and division.

318 There are prominent differences between the models for elongasome and

319 divisome activation, however. The elongasome appears, in essence, to be a stripped down 
320 version of the divisome ${ }^{45}$ : the elongasome contains fewer proteins than the divisome ${ }^{45}$,

321 and when either RodA or PBP2 is hyperactivated through mutation, all elongasome

322 components except MreB and the PG synthases are rendered dispensable ${ }^{5}$. This would

323 suggest that for elongation, the cell needs an activated SEDS-PBP pair and a spatial

324 regulator to orient their motion ${ }^{5}$. Conversely, hyperactive FtsWI in Caulobacter only

325 allows for disruption of FzlA, with the rest of the divisome remaining essential. This may

326 be because division is a more complex process than elongation, requiring invagination

327 and fission of all layers of the envelope in coordination with DNA segregation and cell

328 cycle progression. This complexity necessitates functions in addition to PG synthesis and

329 remodeling provided by components of the divisome. PG synthesis during division likely

330 requires more regulation, as well. Whereas PG synthesis during elongation comprises

331 insertion of new PG in the same plane as old cell wall material, PG synthesis during

332 constriction requires a lasting, directional change to shape the new cell poles. So while

333 there are key similarities in the paradigm of PG synthase activation, regulation of division

334 likely requires a more complicated network of inputs to manage the additional outputs

335 and constraints discussed above. In summation, this work provides evidence that the

336 requirement for SEDS-PBP activation is conserved across multiple modes of PG

337 synthesis, which has broad implications for determining the speed of division, cell shape,

338 and cell wall robustness. 


\section{Methods}

344 Strains, growth conditions, and growth determination

345 Caulobacter crescentus strains were derived from the NA1000 WT strain. Unless

346 otherwise indicated, Caulobacter colonies were isolated from solid 1.5\% agar peptone

347 yeast extract (PYE) plates grown at $30^{\circ} \mathrm{C}$ and cells were grown in liquid culture in PYE

348 shaking at $30{ }^{\circ} \mathrm{C}$. Where indicated, Caulobacter cells were treated with $6 \mu \mathrm{g} / \mathrm{ml}$ of

349 cephalexin. Antibiotic MIC analysis was performed using antibiotic test strips

350 (Liofilchem), which include a concentration gradient of $0.016-256 \mathrm{mg} / \mathrm{L}$ for all

351 antibiotics tested. Where indicated, cells were treated with $0.3 \%$ xylose to drive inducible

352 gene expression. Spot dilutions for Caulobacter were performed by serially diluting cells

353 at the indicated fraction (1/10 or $1 / 2)$, before plating. Growth rates were obtained by

354 measuring optical density at $600 \mathrm{~nm}\left(\mathrm{OD}_{600}\right)$ values of cells every 30 minutes.

355 Caulobacter cell synchrony was performed as previously described ${ }^{12}$. Briefly, log phase

356 cells were washed with $\mathrm{M} 2$ salts $\left(6.1 \mathrm{mM} \mathrm{Na}_{2} \mathrm{HPO}_{4}, 3.9 \mathrm{mM} \mathrm{KH}_{2} \mathrm{PO}_{4}, 9.3 \mathrm{mM} \mathrm{NH} 4 \mathrm{Cl}\right)^{46}$,

357 resuspended in 1:1 M2:Percoll, then centrifuged at 11,200 $\mathrm{x}$. The swarmer band was

358 isolated, and cells were subsequently washed twice in M2, then resuspended in PYE.

359 A. tumefaciens were grown in A. tumefaciens glucose and $\left(\mathrm{NH}_{4}\right)_{2} \mathrm{SO}_{4}(\mathrm{ATGN})$

360 minimal medium ${ }^{47}$, with $0.5 \%$ glucose at $28^{\circ}$ C. E. coli strains were grown in LB medium

361 at $37^{\circ} \mathrm{C}$. IPTG was added at a concentration of $1 \mathrm{mM}$ when necessary. For A. tumefaciens

362 spot dilutions, cells were grown overnight in ATGN minimal medium in the presence of

363 IPTG at $1 \mathrm{mM}$ concentration, washed, then pre-depleted of IPTG for 16 hours where

364 indicated. Cells were then serially diluted (ten-fold) and spotted on ATGN minimal

365 medium with the presence or absence of IPTG. To make the $\Delta f z l A P_{l a c} f z l A$ strain 
366 (PBA199), first, a mini-Tn7 vector containing IPTG inducible $f z l A$, along with the

367 pTNS3 helper plasmid, were introduced into $\Delta t e t R A$ a-att $\operatorname{Tn} 7$ cells (PBA44) via

368 electroporation as previously described ${ }^{48}$. Deletion of $f z l A$ (for PBA199) and allelic

369 exchange of $f t s W$ (for PBA232) were subsequently performed by transferring the

370 corresponding suicide vector to A. tumefaciens via conjugation with E. coli $\mathrm{S} 17$.

$371 \quad$ Plasmids (Supplementary Table 2) and strains (Supplementary Table 3) used

372 in this study can be found in the supplementary information. Plasmids were created using

373 standard molecular cloning procedures including PCR, restriction digestion, and ligation.

374 Mutagenesis of $f$ ts $W$ for $A$. tumefaciens was performed using a QuikChange Lightning

375 Multi Site-Directed Mutagenesis Kit (Agilent Genomics), with primers designed using

376 Agilent's QuikChange Primer Design Program, as previously described ${ }^{22}$. pEG1345 was

377 constructed using an NEBuilder HiFi DNA Assembly Cloning Kit (NEB).

379 Light microscopy imaging and analysis

380 Images of log phase Caulobacter cells were obtained using either phase contrast

381 microscopy, with cells grown on either $1 \%$ agarose PYE pads or $1 \%$ agarose $\mathrm{dH}_{2} \mathrm{O}$ pads,

382 or, when indicated, fluorescence microscopy, with cells grown on $1 \%$ agarose $\mathrm{dH}_{2} \mathrm{O}$

383 pads. For fluorescence microscopy, mNG-FtsZ expression was induced for 1 hour with

384 xylose then imaged through the GFP filter and venus-MreB expression was induced for 2

385 hours with xylose then imaged through the YFP filter. For determination of PG

386 incorporation localization, cells were pulsed with $0.82 \mathrm{mM}$ HADA for 5 minutes, washed

387 twice with PBS, then visualized through the DAPI filter. For time-lapse imaging, as

388 previously described ${ }^{22}$, synchronized cells were placed on $1 \%$ agarose PYE pads and 
389 imaged using phase contrast microscopy at room temperature (RT), with images being

390 acquired at 5 minute intervals at 100x. Imaging of Caulobacter cells was performed using

391 a Nikon Eclipse Ti inverted microscope with a Nikon Plan Fluor $\times 100$ (numeric aperture

392 1.30) oil Ph3 objective and Photometrics CoolSNAP HQ ${ }^{2}$ cooled CCD (charge-coupled

393 device) camera ${ }^{22}$. For $A$. tumefaciens phase contrast microscopy, exponentially growing

394 cells were spotted on $1 \%$ agarose ATGN pads as previously described ${ }^{49}$, then imaged.

395 For A. tumefaciens time-lapse microscopy, images were collected every ten minutes.

396 Microscopy of A. tumefaciens cells was performed with an inverted Nikon Eclipse TiE

397 with a QImaging Rolera em-c ${ }^{2} 1 \mathrm{~K}$ EMCCD camera and Nikon Elements Imaging

398 Software.

399 For determination of dimensions of log phase cells, cell length and width were

400 measured using MicrobeJ software, similar to as previously described ${ }^{22}$. Constriction rate

401 and elongation rate were also determined using Microbe ${ }^{23}$. Briefly, MicrobeJ software

402 allowed for tracking of cells imaged by time-lapse microscopy throughout the division

403 process, with automatic detection of constriction initiation and manual determination of

404 cell separation. Cell length was found for cells at each time point, cell width was found at

405 the site of constriction, and constriction time was calculated by multiplying the number of

406 frames in which constriction was detected by 5 (since images were acquired every 5

407 minutes), allowing for calculation of constriction and elongation rates. Constriction

408 failure rate was determined by counting the number of cells which initiated constriction

409 at one division site, failed, then ultimately divided at a separate site. Prism was used for

410 graphing and statistical analysis of calculated terms. 


\section{Cell shape analysis}

413 For cell shape analysis, binary masks of phase contrast images of log phase cells

414 were inputted into Celltool ${ }^{25}$, allowing for creation of cell contours, similar to as

415 previously described ${ }^{22}$. Following alignment of cell contours (not allowing for reflection),

416 a model of cell shape was created. The shape modes of interest were either plotted as

417 histograms displaying the cell shape across two dimensions, or as single data points. $\mathrm{R}$

418 software was used to perform statistical analyses to compare population variances in

419 shape modes across strains. Prism was used for graphing calculated terms.

420

421 Scanning electron microscopy sample preparation and imaging

422 For SEM, log phase cells were incubated on poly-lysine (1:10) coated glass cover

423 slip for 15 minutes, then fixed for 1 hour using fixation buffer (1\% glutaraldehyde, 0.02

$424 \mathrm{M}$ cacodylate, and $3 \mathrm{mM} \mathrm{MgCl} 2$ ). Cells were gradually dried by washing 3 times with

425 wash buffer ( $3 \%$ sucrose, $0.02 \mathrm{M}$ cacodylate, and $3 \mathrm{mM} \mathrm{MgCl}$ ), twice with $\mathrm{dH}_{2} \mathrm{O}$, once

426 each with $30 \%, 50 \%, 70 \%, 90 \%, 100 \%$ ethanol, once with $1: 1$

427 ethanol:hexamethyldisiloxane (HMDS), and once with HMDS at 5 minute intervals each,

428 before desiccation overnight. Cover slips were mounted, then coated with a $15 \mathrm{~nm}$ gold

429 palladium sputter coat. Samples were then imaged with a LEO/Zeiss Field-emission

430 SEM.

431

432 Immunoblot analysis

433 Immunoblot analysis was performed similar to as previously described ${ }^{22}$, using a

$4341: 5,000-1: 6,666$ dilution of $\alpha$-FzlA primary antibody ${ }^{21}$, a 1:50,000 dilution of $\alpha$-HU 
435 primary antibody ${ }^{50}$, and 1:10,000 of HRP-labeled $\alpha$-rabbit secondary antibody

436 (PerkinElmer) on nitrocellulose membranes. Chemiluminescent substrate (PerkinElmer)

437 was added to facilitate protein visualization via an Amersham Imager 600 RGB gel and

438 membrane imager (GE).

440 Transposon library preparation, sequencing, and analysis

441 Wild type Caulobacter crescentus NA1000 (EG2366) or fts $W^{* *} I^{*}$ triple mutant

442 (EG1557) cells were grown in a large culture (1 liter) to mid-log (0.4-0.6), washed of

443 excess $\mathrm{Mg}^{2+}$ with $10 \%$ glycerol, and mutagenized with the Ez-Tn5 $<$ Kan-2> transposome

444 (Epicentre). Cells recovered by shaking at $30{ }^{\circ} \mathrm{C}$ for 90 minutes, then plated on

445 kanamycin containing plates for 3 days at $30{ }^{\circ} \mathrm{C}$ in order to yield roughly $100-500$

446 colonies per plate. Libraries were grown at $30^{\circ} \mathrm{C}$ and comprised $\sim 100,000-200,000$

447 colonies each. Mutants were pooled into one library by scraping colonies from the

448 surface of the agar and added into 25-40 mL PYE. Pooled libraries were shaken to yield

449 a homogenous slurry and sterile glycerol was added to $20 \%$. Libraries were then frozen in

450 liquid nitrogen and stored at $-80^{\circ} \mathrm{C}$. Two libraries of each genetic background were

451 prepared individually and compared as biological replicates.

452 Genomic DNA was extracted from one aliquot of each library using DNeasy

453 Blood and Tissue Kit (Qiagen). Tn-Seq libraries were prepared for Illumina Next-

454 Generation sequencing through sequential PCR amplifications using arbitrary hexamer

455 primers and Tn5-specific primer facing outward for the first round, and indexing primer

456 sets that include unique molecular identifier to filter artifacts arising from PCR duplicates 
457 for the second round. Libraries were then pooled and sequenced at the University of

458 Massachusetts Amherst Genomics Core Facility on the NextSeq 550 (Illumina).

459 For analyses, reads were demultiplexed by index, then each sample Tn-Seq

460 library was concatenated and clipped of the unique molecular identifier linker from the

461 second PCR using $\mathrm{Je}^{51}$ and the following command:

462 java -jar /je_1.2/je_1.2_bundle.jar clip F1=compiled.gz LEN=6

463 Clipped reads were then mapped back to the Caulobacter NA1000 genome (NCBI

464 Reference Sequence: NC_011916.1) using BWA ${ }^{52}$ and sorted using Samtools ${ }^{53}$ :

465 bwa mem -t2 clipped.gz | samtools sort - - 2 - > sorted.bam

466 Duplicate reads were removed using $\mathrm{Je}^{51}$ and indexed with Samtools ${ }^{53}$ using the following

467 command:

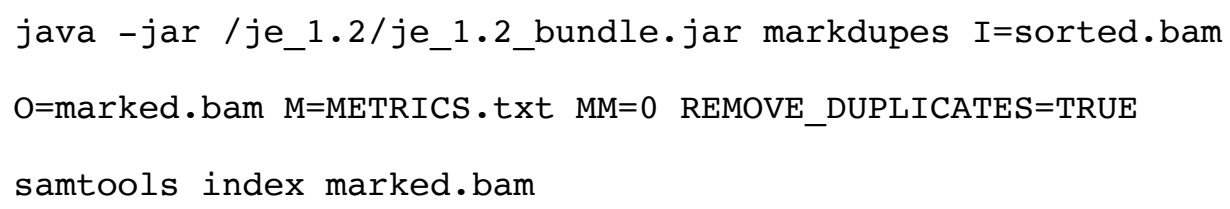

471

4725 ' sites of inserted transposons from each library were converted into .wig files

473 containing counts per position and viewed using Integrative Genomics Viewer ${ }^{54,55}$.

474 Coverage and insertion frequency using a bedfile containing all open reading frames from

475 NC_011916.1 with the outer 20\% of each gene removed were determined using

476 BEDTools ${ }^{56}$ and the following commands:

477 bedtools genomecov -5 -bg marked.bam $>$ marked.bed

478 bedtools map -a NA1000.txt -b marked.bed -c $4>$ output.txt

479 
bioRxiv preprint doi: https://doi.org/10.1101/453415; this version posted October 25, 2018. The copyright holder for this preprint (which was not certified by peer review) is the author/funder, who has granted bioRxiv a license to display the preprint in perpetuity. It is made available under aCC-BY 4.0 International license.

480 Comparison of transposon insertions was performed using the edgeR package in the

481 Bioconductor suite ${ }^{57,58}$ using a quasi-likelihood F-test (glmQLFit) to determine the false

482 discovery rate adjusted $\mathrm{p}$-values reported here.

483

484

485

486

487

488

489

490

491

492

493

494

495

496 


\section{References}

498 1. Cabeen, M. T. \& Jacobs-Wagner, C. Bacterial cell shape. Nat. Rev. Microbiol. 3, $499 \quad 601-610(2005)$

500 2. Woldemeskel, S. A. \& Goley, E. D. Shapeshifting to Survive: Shape Determination 501 and Regulation in Caulobacter crescentus. Trends Microbiol. 25, 673-687 (2017).

502 3. Gan, L., Chen, S. \& Jensen, G. J. Molecular organization of Gram-negative 503 peptidoglycan. Proc. Natl. Acad. Sci. 105, 18953-18957 (2008).

504 4. Huang, K. C., Mukhopadhyay, R., Wen, B., Gitai, Z. \& Wingreen, N. S. Cell shape 505 and cell-wall organization in Gram-negative bacteria. Proc. Natl. Acad. Sci. 506 pnas.0805309105 (2008). doi:10.1073/pnas.0805309105

507 5. Rohs, P. D. A. et al. A central role for PBP2 in the activation of peptidoglycan 508 polymerization by the bacterial cell elongation machinery. PLOS Genet. 14, 509 e1007726 (2018).

510 6. Meeske, A. J. et al. SEDS proteins are a widespread family of bacterial cell wall 511 polymerases. Nature 537, 634-638 (2016).

512 7. Taguchi, A. et al. FtsW is a peptidoglycan polymerase that is activated by its cognate 513 penicillin-binding protein. bioRxiv 358663 (2018). doi:10.1101/358663

514 8. Lambert, A. et al. Constriction Rate Modulation Can Drive Cell Size Control and 515 Homeostasis in C. crescentus. iScience 4, 180-189 (2018).

516 9. Modell, J. W., Kambara, T. K., Perchuk, B. S. \& Laub, M. T. A DNA Damage517 Induced, SOS-Independent Checkpoint Regulates Cell Division in Caulobacter 518 crescentus. PLOS Biol 12, e1001977 (2014). 
519 10. Vaughan, S., Wickstead, B., Gull, K. \& Addinall, S. G. Molecular evolution of FtsZ

520 protein sequences encoded within the genomes of archaea, bacteria, and eukaryota. $J$.

$521 \quad$ Mol. Evol. 58, 19-29 (2004).

522 11. Sundararajan, K. \& Goley, E. D. Cytoskeletal Proteins in Caulobacter crescentus:

523 Spatial Orchestrators of Cell Cycle Progression, Development, and Cell Shape. in

524 Prokaryotic Cytoskeletons 103-137 (Springer, Cham, 2017). doi:10.1007/978-3-319-

$525 \quad 53047-5 \_4$

526 12. Goley, E. D. et al. Assembly of the Caulobacter cell division machine. Mol.

$527 \quad$ Microbiol. 80, 1680-1698 (2011).

528 13. Holden, S. J. et al. High throughput 3D super-resolution microscopy reveals

529 Caulobacter crescentus in vivo Z-ring organization. Proc. Natl. Acad. Sci. 111, 4566-

$530 \quad 4571(2014)$.

531 14. Fu, G. et al. In vivo structure of the E. coli FtsZ-ring revealed by photoactivated

532 localization microscopy (PALM). PloS One 5, e12682 (2010).

533 15. Buske, P. J. \& Levin, P. A. A flexible C-terminal linker is required for proper FtsZ

$534 \quad$ assembly in vitro and cytokinetic ring formation in vivo. Mol. Microbiol. 89, 249-

$535263(2013)$

536 16. Sundararajan, K. et al. The bacterial tubulin FtsZ requires its intrinsically disordered

537 linker to direct robust cell wall construction. Nat. Commun. 6, 7281 (2015).

538 17. Gardner, K. A. J. A., Moore, D. A. \& Erickson, H. P. The C-terminal linker of

539 Escherichia coli FtsZ functions as an intrinsically disordered peptide. Mol. Microbiol.

$540 \quad$ 89, 264-275 (2013). 
541 18. Howell, M. L. et al. Agrobacterium tumefaciens divisome proteins regulate the

542 transition from polar growth to cell division. bioRxiv 412759 (2018).

543 doi: $10.1101 / 412759$

544 19. Yang, X. et al. GTPase activity-coupled treadmilling of the bacterial tubulin FtsZ

$545 \quad$ organizes septal cell wall synthesis. Science 355, 744-747 (2017).

546 20. Bisson-Filho, A. W. et al. Treadmilling by FtsZ filaments drives peptidoglycan

547 synthesis and bacterial cell division. Science 355, 739-743 (2017).

548 21. Goley, E. D., Dye, N. A., Werner, J. N., Gitai, Z. \& Shapiro, L. Imaging-based

549 identification of a critical regulator of FtsZ protofilament curvature in Caulobacter.

$550 \quad$ Mol. Cell 39, 975-987 (2010).

551 22. Lariviere, P. J., Szwedziak, P., Mahone, C. R., Löwe, J. \& Goley, E. D. FzlA, an

552 essential regulator of FtsZ filament curvature, controls constriction rate during

553 Caulobacter division. Mol. Microbiol. 107, 180-197 (2018).

554 23. Ducret, A., Quardokus, E. M. \& Brun, Y. V. MicrobeJ, a tool for high throughput

555 bacterial cell detection and quantitative analysis. Nat. Microbiol. 1, 16077 (2016).

556 24. Coltharp, C., Buss, J., Plumer, T. M. \& Xiao, J. Defining the rate-limiting processes

557 of bacterial cytokinesis. Proc. Natl. Acad. Sci. 113, E1044-E1053 (2016).

558 25. Pincus, Z. \& Theriot, J. A. Comparison of quantitative methods for cell-shape

559 analysis. J. Microsc. 227, 140-156 (2007).

560 26. Charbon, G., Cabeen, M. T. \& Jacobs-Wagner, C. Bacterial intermediate filaments: in

561 vivo assembly, organization, and dynamics of crescentin. Genes Dev. 23, 1131-1144

$562 \quad$ (2009). 
563 27. Kuru, E. et al. In situ Probing of Newly Synthesized Peptidoglycan in Live Bacteria

564 with Fluorescent D-Amino Acids. Angew. Chem. Int. Ed Engl. 51, 12519-12523

565 (2012).

566 28. Pogliano, J., Pogliano, K., Weiss, D. S., Losick, R. \& Beckwith, J. Inactivation of

567 FtsI inhibits constriction of the FtsZ cytokinetic ring and delays the assembly of FtsZ

568 rings at potential division sites. Proc. Natl. Acad. Sci. U. S. A. 94, 559-564 (1997).

569 29. Costa, T., Priyadarshini, R. \& Jacobs-Wagner, C. Localization of PBP3 in

570 Caulobacter crescentus is highly dynamic and largely relies on its functional

571 transpeptidase domain. Mol. Microbiol. 70, 634-651 (2008).

572 30. Spratt, B. G. The mechanism of action of mecillinam. J. Antimicrob. Chemother. 3,

$573 \quad 13-19(1977)$.

574 31. Spratt, B. G. \& Pardee, A. B. Penicillin-binding proteins and cell shape in E. coli.

$575 \quad$ Nature 254, 516-517 (1975).

576 32. Rechenberg, M. von et al. Ampicillin/penicillin-binding protein interactions as a

577 model drug-target system to optimize affinity pull-down and mass spectrometric

578 strategies for target and pathway identification. PROTEOMICS 5, 1764-1773 (2005).

579 33. Reynolds, P. E. Structure, biochemistry and mechanism of action of glycopeptide

580 antibiotics. Eur. J. Clin. Microbiol. Infect. Dis. 8, 943-950 (1989).

581 34. Michalopoulos, A. S., Livaditis, I. G. \& Gougoutas, V. The revival of fosfomycin.

582 Int. J. Infect. Dis. 15, e732-e739 (2011).

583 35. Yakhnina, A. A. \& Gitai, Z. Diverse Functions for Six Glycosyltransferases in

584 Caulobacter crescentus Cell Wall Assembly. J. Bacteriol. 195, 4527-4535 (2013). 
585 36. Strobel, W., Möll, A., Kiekebusch, D., Klein, K. E. \& Thanbichler, M. Function and

586 Localization Dynamics of Bifunctional Penicillin-Binding Proteins in Caulobacter

587 crescentus. J. Bacteriol. 196, 1627-1639 (2014).

588 37. Meier, E. L. et al. FtsEX-mediated regulation of the final stages of cell division

589 reveals morphogenetic plasticity in Caulobacter crescentus. PLOS Genet. 13,

$590 \quad$ e1006999 (2017).

591 38. Goley, E. D., Comolli, L. R., Fero, K. E., Downing, K. H. \& Shapiro, L. DipM links

592 peptidoglycan remodelling to outer membrane organization in Caulobacter. Mol.

$593 \quad$ Microbiol. 77, 56-73 (2010).

594 39. Möll, A., Schlimpert, S., Briegel, A., Jensen, G. J. \& Thanbichler, M. DipM, a new

595 factor required for peptidoglycan remodelling during cell division in Caulobacter

596 crescentus. Mol. Microbiol. 77, 90-107 (2010).

597 40. Zielińska, A. et al. LytM factors affect the recruitment of autolysins to the cell

598 division site in Caulobacter crescentus. Mol. Microbiol. 106, 419-438 (2017).

599 41. Zuleta, L. F. G., Italiani, V. C. S. \& Marques, M. V. Isolation and Characterization of

$600 \quad \mathrm{NaCl}-$ Sensitive Mutants of Caulobacter crescentus. Appl Env. Microbiol 69, 3029-

$6013035(2003)$.

602 42. Aaron, M. et al. The tubulin homologue FtsZ contributes to cell elongation by

603 guiding cell wall precursor synthesis in Caulobacter crescentus. Mol. Microbiol. 64, $604 \quad 938-952(2007)$.

605 43. Brown, P. J. B. et al. Polar growth in the Alphaproteobacterial order Rhizobiales.

606 Proc. Natl. Acad. Sci. 109, 1697-1701 (2012). 
607 44. Figueroa-Cuilan, W. M. \& Brown, P. J. B. Cell Wall Biogenesis During Elongation

608 and Division in the Plant Pathogen Agrobacterium tumefaciens. Curr. Top.

609 Microbiol. Immunol. (2018). doi:10.1007/82_2018_92

610 45. Szwedziak, P. \& Löwe, J. Do the divisome and elongasome share a common

611 evolutionary past? Curr. Opin. Microbiol. 16, 745-751 (2013).

612 46. Hottes, A. K. et al. Transcriptional Profiling of Caulobacter crescentus during

613 Growth on Complex and Minimal Media. J. Bacteriol. 186, 1448-1461 (2004).

614 47. Morton, E. R. \& Fuqua, C. UNIT 3D.1 Laboratory Maintenance of Agrobacterium.

615 Curr. Protoc. Microbiol. CHAPTER, Unit3D.1 (2012).

616 48. Figueroa-Cuilan, W., Daniel, J. J., Howell, M., Sulaiman, A. \& Brown, P. J. B. Mini-

617 Tn7 Insertion in an Artificial attTn7 Site Enables Depletion of the Essential Master

618 Regulator CtrA in the Phytopathogen Agrobacterium tumefaciens. Appl. Environ.

$619 \quad$ Microbiol. 82, 5015-5025 (2016).

620 49. Howell, M., Daniel, J. J. \& Brown, P. J. B. Live Cell Fluorescence Microscopy to

621 Observe Essential Processes During Microbial Cell Growth. J. Vis. Exp. JoVE

$622 \quad$ (2017). doi:10.3791/56497

623 50. Bowman, G. R. et al. Caulobacter PopZ forms a polar subdomain dictating sequential

624 changes in pole composition and function. Mol. Microbiol. 76, 173-189 (2010).

625 51. Girardot, C., Scholtalbers, J., Sauer, S., Su, S.-Y. \& Furlong, E. E. M. Je, a versatile

626 suite to handle multiplexed NGS libraries with unique molecular identifiers. $B M C$

627 Bioinformatics 17, 419 (2016).

628 52. Li, H. \& Durbin, R. Fast and accurate long-read alignment with Burrows-Wheeler

629 transform. Bioinforma. Oxf. Engl. 26, 589-595 (2010). 
630 53. Li, H. et al. The Sequence Alignment/Map format and SAMtools. Bioinforma. Oxf.

$631 \quad$ Engl. 25, 2078-2079 (2009).

632 54. Robinson, J. T. et al. Integrative genomics viewer. Nat. Biotechnol. 29, 24-26 (2011).

633 55. Thorvaldsdóttir, H., Robinson, J. T. \& Mesirov, J. P. Integrative Genomics Viewer

634 (IGV): high-performance genomics data visualization and exploration. Brief.

635 Bioinform. 14, 178-192 (2013).

636 56. Quinlan, A. R. \& Hall, I. M. BEDTools: a flexible suite of utilities for comparing

637 genomic features. Bioinformatics 26, 841-842 (2010).

638 57. Robinson, M. D., McCarthy, D. J. \& Smyth, G. K. edgeR: a Bioconductor package

639 for differential expression analysis of digital gene expression data. Bioinforma. Oxf.

$640 \quad$ Engl. 26, 139-140 (2010).

641 58. McCarthy, D. J., Chen, Y. \& Smyth, G. K. Differential expression analysis of

642 multifactor RNA-Seq experiments with respect to biological variation. Nucleic Acids

$643 \quad$ Res. 40, 4288-4297 (2012).

644

645

646

647

648

649

650

651

652 


\section{Acknowledgements}

654 We would like to thank Josh Modell and Mike Laub for providing strains; the Manley

655 lab, especially Ambroise Lambert, and members of the Xiao and Goley labs for helpful

656 discussions; the Goley lab for feedback on the manuscript; Anant Bhargava and Adrien

657 Ducret for help with time-lapse analysis; Mike Delannoy, Barbara Smith, and Selam

658 Woldemeskel for developing the SEM protocol, and Mike and Barbara for training on

659 SEM equipment; and Brandon King for providing support with experiments. This work

660 funded in part by the National Institutes of Health, National Institute of General Medical

661 Sciences through R01GM108640 (EDG and PJL), T32GM007445 (training grant support

662 of PJL), R01GM111706 (PC and RZ), R25GM056901(training support of GS-C), and

663 T32GM08515 (training grant support of RZ). PB and MH were supported by the National

664 Science Foundation, IOS1557806.

\section{Author Information}

666 PJL, CRM, AKD, PB, MH, GS-C, PC, RZ, and EDG planned the experiments, PJL,

667 CRM, AKD, MH, GS-C, and RZ performed the experiments, PJL, CRM, AKD, MH, and

668 EDG wrote the manuscript, and PJL, CRM, AKD, PB, MH, GS-C, PC, and EDG edited

669 the manuscript.

670

671 Competing Interests

672 The authors declare no competing interests

673

674

675 


\section{$676 \quad$ Figure legends}

680 A. Phase contrast microscopy images depicting WT Caulobacter and PG synthase

681 hyperactive mutant cells with and without $f z l A$. White asterisks mark S-shaped cells.

682 Scale bar $=2 \mu \mathrm{m}$.

683 B. $\alpha$-FzlA immunoblot (top) and $\alpha$-HU immunoblot (bottom, loading control) of the

684 indicated strains.

685 C, D. Spot dilutions (ten-fold serial dilutions) (C) and growth curves (D) of the indicated 686 strains.

687 E. Lengths of unsynchronized cells from the indicated strains. Mean \pm SEM shown.

688 Kruskal-Wallis tests with Dunn's post-test were performed to analyze differences

689 compared to WT and the indicated strains: ${ }^{* * *} P \leq 0.001$. From left to right, $n=254,262$,

$690 \quad 261,260,258$.

691 F. Volcano plot of the negative $\log _{10}$ of the false discovery rate $(-\log (\mathrm{FDR}))$ vs. $\log _{2}$ of

692 the fold change of each gene in WT vs. $f t s W^{* *} I^{*}$ strains determined by Tn-Seq analysis.

693 G. Plot of transposon insertion frequency in essential division genes in WT (top) vs.

$694 f t W^{* *} I^{*}$ (bottom) cells determined by Tn-Seq analysis. Genetic loci are annotated below

695 the plot. Number of reads is displayed on a logarithmic scale.

696 Strain key (Caulobacter crescentus): WT (EG865 A-E; EG2366 F-G), fts $W^{* *} I^{*}$

697 (EG1557), fts $W^{* *} I^{*} \Delta f z l A(\mathrm{EG} 2170), f t s W^{*}(\mathrm{EG} 1556), f t s W^{*} \Delta f z l A(\mathrm{EG} 2166)$. 


\section{Fig. 2: FzlA contributes to efficient division in a hyperactive PG synthase}

\section{1 background}

702 A. Phase contrast time-lapse microscopy images depicting constriction in WT or PG

703 synthase hyperactive mutant cells with and without $f z l A$. Constriction starts at $\mathrm{t}=0$ and

704 concludes in the last frame upon cell separation. Time relative to constriction initiation

705 (minutes) is indicated. Scale bar $=1 \mu \mathrm{m}$.

$706 \mathrm{~B}, \mathrm{C}, \mathrm{D}$. Plots of constriction rate (B), total elongation rate $(\mathrm{C})$, and ratio of constriction

707 rate to total elongation rate (D) for a population of synchronized cells from each indicated

708 strain, calculated from single cell microscopy data. Mean $\pm \mathrm{SEM}$ shown. Kruskal-Wallis

709 tests with Dunn's post-test were performed to analyze differences compared to WT and

710 the indicated strains: ${ }^{* * *} P \leq 0.001$. From left to right, $n=324,280,161,366,139(\mathrm{~B})$ and

$711321,280,161,363,139(\mathrm{C}, \mathrm{D})$.

712 E. Phase contrast microscopy images depicting constriction failure at the initial division

713 site (single white arrowhead), then initiation and completion at a second site (double

714 white arrowhead) in $\Delta f z l A$ cells. As in (A), constriction initiates at $\mathrm{t}=0$ and concludes in

715 the last frame. Scale bar $=1 \mu \mathrm{m}$.

716 F. Plot of the constriction failure rate in cells in which constriction initiated, then failed at

717 one division site and subsequently initiated and finished at another division site. "Number

718 of aborted division events" refers to the number of times a cell abandoned division at

719 distinct sites within the cell. The y-axis indicates the percentage of cells out of the whole

720 population that displayed at least one such aborted division event. From left to right, $n=$

$721324,280,193,368,174$. 
722 Strain key (Caulobacter crescentus): WT (EG865), fts $W^{* *} I^{*}(\mathrm{EG} 1557), f t s W^{* *} I^{*} \Delta f z l A$

723 (EG2170), fts $W^{*}(\mathrm{EG} 1556), f t s W^{*} \Delta f z l A(\mathrm{EG} 2166)$.

\section{Fig. 3: $f z l A$ is required for global shape maintenance}

726 A. SEM images of cells from the indicated strains. Scale bar $=200 \mathrm{~nm}$.

727 B. PCA of cell shape in a population of cells that have initiated constriction from the

728 indicated strains. Shape mode 3 approximately captures degree of S-shape in cells. Mean

729 cell contour \pm 1 or 2 standard deviations (s.d.) is shown (left). Shape mode values for

730 cells in each strain are plotted and mean \pm SEM is shown (right). The dashed line drawn

731 at s.d. $=1$ indicates the cutoff for S-shaped cells (cells with an $\mid$ s.d. $\mid \geq 1$ are considered to

732 be S-shaped). A Brown-Forsythe Levene-type test (which is used in populations not

733 assumed to be normally distributed) was performed to determine differences between

734 population variances $(\dagger):{ }^{n s} P>0.05,{ }^{* * *} P \leq 0.001$. From left to right, $n=292,279,290$.

735 C. Plot of shape mode 3 (S-shape) vs. shape mode 1 (length) values for a population of

736 cells that have initiated constriction from the indicated strains. The dashed lines drawn at

737 s.d. $\mid=1$ indicates the cutoff for S-shaped cells.

738 D. Plot of percentage of S-shaped cells present in a population of cells that have initiated

739 constriction from the indicated strains. Cells from (B) with an $\mid$ s.d. $\mid \geq 1$ are considered to

740 be S-shaped.

741 E. Phase contrast microscopy images depicting cell twisting during division in an

$742 f$ ts $^{* *} I^{*} \Delta f z l A$ cell. Constriction starts at $\mathrm{t}=0$ minutes and concludes in the last frame

743 upon cell separation. Scale bar $=1 \mu \mathrm{m}$. 
744 Strain key (Caulobacter crescentus): WT (EG865), fts $W^{* *} I^{*}(\mathrm{EG} 1557), f t s W^{* *} I^{*} \Delta f z l A$

745 (EG2170).

746

747 Fig. 4: Loss of $\boldsymbol{f} \boldsymbol{z} \boldsymbol{l A}$ leads to increased cell wall antibiotic sensitivity

748 A. Spot dilutions (diluted ten-fold) of the indicated strains plated on PYE \pm cephalexin (6

$749 \mu \mathrm{g} / \mathrm{ml})$.

750 B. Plates of the indicated strains grown in the presence of mecillinam minimum

751 inhibitory concentration (MIC) test strips, with antibiotic concentration decreasing from

752 top to bottom. The zone of clearance is highlighted in white (dashed line).

753 Strain key (Caulobacter crescentus): WT (EG865), fts $W^{* *} I^{*}(\mathrm{EG} 1557), f t s W^{* *} I^{*} \Delta f z l A$

754 (EG2170).

755

756 Fig. 5: The ability of hyperactive $f t s W$ to suppress loss of $f z l A$ is conserved

757 A. Phase contrast microscopy images depicting PG synthase hyperactive mutant cells \pm

758 FzlA in Agrobacterium tumefaciens. FzlA was induced where indicated with IPTG and

759 depleted where indicated upon removal of IPTG, then grown for 16 hours on agarose

760 pads. White arrowheads mark ectopic poles at midcell. Scale bar $=2 \mu \mathrm{m}$.

761 B. Spot dilutions (ten-fold serial dilutions) of the indicated strains grown in the presence

762 or absence of IPTG to control fzlA expression, in A. tumefaciens.

763 Strain key (A. tumefaciens): WT (PBA44) $)^{48} ; \Delta f z l A P_{l a c} f z l A(\mathrm{PBA} 199) ; \Delta f z l A P_{l a c} f z l A$

764 ftsWF137L (PBA232).

765 


\section{Fig. 6: FzlA is required for activation of FtsWI and regulates the geometry of PG}

\section{7 insertion}

768 FzlA is required for activation of FtsWI and likely signals through unidentified

769 intermediate factor(s) in a manner dependent on interaction with FtsZ to effect normal

770 cell shape, normal constriction rate, and antibiotic resistance (left). FtsW**I* can still

771 receive input from FzlA, which in combination with its own hyperactivity, leads to faster

772 constriction and antibiotic sensitivity likely associated with positive misregulation of PG

773 insertion (middle). FtsW**I* can function in the absence of FzlA, but with misregulated

774 activity, leading to twisting during constriction, slower constriction speed, and increased

775 antibiotic sensitivity (right).

776

777 
1 Activation of SEDS-PBP cell wall synthases by an essential regulator of bacterial

2 division

3

4 Lariviere et al

5

6 Supplementary Information

8 Supplementary Figure Legends

$9 \quad$ Fig. S1: Uncropped Immunoblots

10 A-C. Uncropped $\alpha$-FzlA immunoblots (top) and $\alpha$-HU immunoblots (bottom, loading

11 control) of the indicated strains from figure 1 (A), figure S2 (B, left half), and figure S3

12 (C).

13

14 Fig. S2: fts $W^{* *} I *$ rescue the fitness/morphological defects of two $f z l A$ mutants

15 A. Phase contrast microscopy images depicting cells of the indicated strains. Scale bar $=$ $162 \mu \mathrm{m}$.

17 B, C. Spot dilutions (diluted ten-fold) (B) and growth curves (C) of the indicated strains.

18 D. $\alpha$-FzlA immunoblot (top) and $\alpha$-HU immunoblot (bottom, loading control) of the

19 indicated strains.

20 E, F. Lengths (E) and widths (F) of unsynchronized cells from the indicated strains. Mean

$21 \pm$ SEM shown. Kruskal-Wallis tests with Dunn's post-test were performed to analyze

22 differences compared to the indicated strains: ${ }^{* * *} P \leq 0.001$. From left to right, $n=674$,

$23609,606,653,618,645$.

24 Strain key (Caulobacter crescentus): WT (EG865), fts $W^{* *} I^{*} f z l A(\mathrm{EG} 1557), f z l A^{N H 2}$

25 (EG1600), fts $W^{* *} I^{*} f z l A^{N H 2}$ (EG2111), fzl $A^{N H 3}$ (EG1909), fts $W^{* *} I^{*} f z l A^{N H 3}$ (EG2489). 
27 Fig. S3: In the presence of hyperactive PG synthases, the interaction between FtsZ

28 and FzlA determines division efficiency, but not growth rate or viability

29 A. Phase contrast microscopy images depicting cells of the indicated strains. White

30 asterisks mark S-shaped cells. Scale bar $=2 \mu \mathrm{m}$.

31 B. $\alpha$-FzlA immunoblot (top) and $\alpha-\mathrm{HU}$ immunoblot (bottom, loading control) of the

32 indicated strains.

33 C, D. Spot dilutions (diluted ten-fold) (C) and growth curves (D) of the indicated strains.

34 E. Lengths of unsynchronized cells from the indicated strains. Mean \pm SEM shown.

35 Kruskal-Wallis tests with Dunn's post-test were performed to analyze differences

36 compared to WT and the indicated strains: ${ }^{n s} P>0.05,{ }^{* * *} P \leq 0.001$. From left to right, $n=$

$37609,653,645,688,674,729,612$.

38 Strain key (Caulobacter crescentus): fts $W^{* *} I^{*} f z l A(\mathrm{EG} 1557), f t s W^{* *} I^{*} f z l A^{N H 2}$

39 (EG2111), fts $W^{* *} I^{*} f z l A^{N H 3}(\mathrm{EG} 2489), f t s W^{* *} I^{*} f z l A^{N H 1}(\mathrm{EG} 2492), f t s W^{* *} I * f z l A^{N B 2}$

$40 \quad(\mathrm{EG} 2485), f t s W^{* *} I^{*} f z l A^{N B 1}(\mathrm{EG} 2495), f t s W^{* *} I^{*} \Delta f z l A(\mathrm{EG} 2170)$.

42 Fig. S4: FtsZ localization is unaffected in $f t s W^{* *} I * \Delta f z l A$ cells

43 Phase contrast, fluorescence, and merged microscopy images depicting mNG-FtsZ

44 localization in cells of the indicated strains in the presence of inducer (xylose). White

45 asterisks mark S-shaped cells. Scale bar $=2 \mu \mathrm{m}$.

46 Strain key (Caulobacter crescentus): fts $W^{* *} I^{*}+p X y l m N G-f t s Z(\mathrm{EG} 2157), f t s W^{* *} I^{*}$

$47 \Delta f z l A+p X y l m N G-f t s Z(\mathrm{EG} 2326)$.

48 


\section{Fig. S5: MreB localization is unaffected in $f t s W^{* *} I * \Delta f z l A$ cells}

51 Phase contrast, fluorescence, and merged microscopy images depicting Venus-MreB

52 localization in cells of the indicated strains in the presence of inducer (xylose). White

53 asterisks mark S-shaped cells. Scale bar $=2 \mu \mathrm{m}$.

54 Strain key (Caulobacter crescentus): $f t s W^{* *} I^{*}+p X y l$ Venus-mreB (EG2377), fts $W^{* *} I^{*}$

$55 \Delta f z l A+p X y l$ Venus-mreB (EG2378).

57 Fig. S6: The localization of $\mathrm{PG}$ synthesis is unaffected in $f t s W^{* *} I * \Delta f z l A$ cells

58 Phase contrast, fluorescence, and merged microscopy images depicting HADA

59 localization in cells of the indicated strains after a 5 minute HADA pulse. White asterisks

60 mark S-shaped cells. Scale bar $=2 \mu \mathrm{m}$.

61 Strain key (Caulobacter crescentus): WT (EG865), fts $W^{* *} I^{*}(\mathrm{EG} 1557), f t s W^{* *} I^{*} \Delta f z l A$

62 (EG2170).

64 Fig. S7: Interaction of FtsZ with FzlA is necessary for proper division site shape

\section{5 maintenance}

66 A. PCA of cell shape in a population of unsynchronized cells (not all cells are necessarily

67 actively constricting) from the indicated strains. Shape mode 3 approximately captures

68 degree of S-shape in cells. Mean cell contour \pm 1 or 2 standard deviations (s.d.) is shown

69 (left). Shape mode values for cells in each strain are plotted and mean $\pm \mathrm{SEM}$ is shown

70 (right). A Brown-Forsythe Levene-type test (which is used in populations not assumed to

71 be normally distributed) was performed to determine differences between population 
72 variances $(\dagger):{ }^{n s} P>0.05,{ }^{* * *} P \leq 0.001$. From left to right, $n=269,375,218,250,177,289$,

73211.

74 B. Plot of shape mode 3 (degree of S-shape) vs. shape mode 1 (length) values in a

75 population of unsynchronized cells (not all cells are necessarily actively constricting)

76 from the indicated strains. The dashed lines qualitatively demark the boundary between

77 cells that appear to be S-shaped and those that display normal curvature. Inset presents a

78 zoomed in view of the highlighted region of interest.

79 Strain key (Caulobacter crescentus): fts $W^{* *} I^{*} f z l A(\mathrm{EG} 1557), f t s W^{* *} I^{*} f z l A^{N H 2}$

80 (EG2111), fts $W^{* *} I^{*} f z l A^{N H 3}(\mathrm{EG} 2489), f t s W^{* *} I^{*} f z l A^{N H 1}(\mathrm{EG} 2492), f t s W^{* *} I^{*} f z l A^{N B 2}$

81 (EG2485), fts $W^{* *} I^{*} f z l A^{N B 1}(\mathrm{EG} 2495), f t s W^{* *} I^{*} \Delta f z l A(\mathrm{EG} 2170)$.

82

83 Fig. S8: Loss of $\boldsymbol{f} \boldsymbol{z} \boldsymbol{l A}$ does not confer increased sensitivity to various classes of

84 antibiotics

85 Plates of the indicated strains grown in the presence of antibiotic minimum inhibitory

86 concentration (MIC) test strips, with antibiotic concentration decreasing from top to

87 bottom.

88 Strain key (Caulobacter crescentus): WT (EG865), fts $W^{* *} I^{*}(\mathrm{EG} 1557), f t s W^{* *} I^{*} \Delta f z l A$

89 (EG2170).

90

91 Fig. S9: Interaction of FtsZ with FzlA is necessary for increased resistance to cell

92 wall antibiotics

93 Spot dilutions (diluted two-fold) of the indicated strains plated on PYE \pm cephalexin (6

$94 \mu \mathrm{g} / \mathrm{ml})$. 
95 Strain key (Caulobacter crescentus): fts $W^{* *} I^{*} f z l A(\mathrm{EG} 1557), f t s W^{* *} I^{*} f z l A^{N H 2}$

96 (EG2111), fts $W^{* *} I^{*} f z l A^{N H 3}(\mathrm{EG} 2489), f t s W^{* *} I^{*} f z l A^{N H 1}(\mathrm{EG} 2492), f t s W^{* *} I^{*} f z l A^{N B 2}$

97 (EG2485), fts $W^{* *} I^{*} f z l A^{N B 1}(\mathrm{EG} 2495), f t s W^{* *} I^{*} \Delta f z l A(\mathrm{EG} 2170), f z l A^{N H 2}(\mathrm{EG} 1600)$,

$98 f z l A^{N H 3}$ (EG1909).

99

100 Fig. S10: Multiple non-essential division genes become essential in a hyperactive PG

101 synthase background

102 A. Volcano plot of the negative $\log _{10}$ of the false discovery rate $(-\log (\mathrm{FDR})) \mathrm{vs} \log _{2}$ of

103 the fold change of each gene in WT vs. fts $W^{* *} I^{*}$ strains determined by Tn-Seq analysis.

104 This is a zoomed in and cropped view of the volcano plot from Figure 1F.

105 B. Plot of transposon insertion frequency in essential division genes in WT (top) vs.

$106 \mathrm{fts}^{* *} \mathrm{I}^{*}$ (bottom) cells. Genetic loci are annotated below the plot. Number of reads is

107 displayed on a logarithmic scale.

108 Strain key (Caulobacter crescentus): WT (EG2366), fts $W^{* *} I^{*}(\mathrm{EG} 1557)$

109

110 Fig. S11: Time-lapse showing hyperactive $f t s W$ suppresses loss of $f z l A$ in $A$.

111 tumafaciens

112 Phase contrast time-lapse microscopy images depicting WT and PG synthase hyperactive

113 mutant cells depleted of FzlA over time. White arrowheads mark ectopic poles at midcell.

114 Scale bar $=2 \mu \mathrm{m}$.

115 Strain key (Agrobacterium tumefaciens): $\Delta f z l A P_{l a c} f z l A$ (PBA199); $\Delta f z l A P_{l a c} f z l A$

116 ftsWF137L (PBA232). 


\section{Supplementary Video Legends}

\section{Supplementary Video 1:}

121 Phase contrast time-lapse microscopy movies depicting division in WT or PG synthase

122 hyperactive mutant cells with and without $f z l A$. As indicated, 5 minutes elapse between

123 frames. Video playback is 10 frames per second.

124 Strain key (Caulobacter crescentus): WT (EG865), fts $W^{* *} I^{*}(\mathrm{EG} 1557), f t s W^{* *} I^{*} \Delta f z l A$

125 (EG2170), fts $W^{*}(\mathrm{EG} 1556), f t s W^{*} \Delta f z l A(\mathrm{EG} 2166)$.

127 Supplementary Video 2:

128 Phase contrast time-lapse microscopy movies depicting examples of constriction failure

129 at the initial division site then initiation and completion at a subsequent site in $\Delta f z l A$ cells.

130 As indicated, 5 minutes elapse between frames. Video playback is 10 frames per second.

131 Strain key (Caulobacter crescentus): fts $W^{* *} I^{*} \Delta f z l A(\mathrm{EG} 2170), f t s W^{*} \Delta f z l A(\mathrm{EG} 2166)$.

\section{Supplementary Video 3:}

134 Phase contrast microscopy movies depicting examples of cell twisting during division in

135 multiple $f t s W^{* *} I^{*} \Delta f z l A$ cells. As indicated, 5 minutes elapse between frames. Video

136 playback is 10 frames per second. Strain key (Caulobacter crescentus): fts $W^{* *} I^{*} \Delta f z l A$

137 (EG2170). 


\section{Supplementary Tables}

\section{Supplementary Table 1:}

143 Tn-Seq data and analysis for WT vs. $f t s W^{* *} I^{*}$ Caulobacter genes. Columns WT 1, WT 2,

144 WI 1, and WI 2 contain the number of unique transposon insertions in each gene in each

145 replicate of WT or $f t W^{* *} I^{*}$ (WI) strain transposon insertion library. These values were

146 used to determine the $\log _{2}$ fold-change $(\log \mathrm{FC})$, $\log$ counts per million reads $(\log \mathrm{CPM})$,

147 PValue, false-discovery rate (FDR), and negative $\log _{10}$ of the FDR for each gene in WT

148 versus $f t s W^{* *} I^{*}$. Genes are ordered by significance (neglog(FDR)).

150 Supplementary Table 2:

151 List of plasmids used in this study.

\section{Supplementary Table 3:}

154 List of strains used in this study. 


\section{Supplementary References}

165

166

167

168

169

170

171

172

173

174

175

176

177

178

179

180

181

182

183

184

185

186

187

1. Goley, E. D., Dye, N. A., Werner, J. N., Gitai, Z. \& Shapiro, L. Imaging-based identification of a critical regulator of FtsZ protofilament curvature in Caulobacter. Mol. Cell 39, 975-987 (2010).

2. Lariviere, P. J., Szwedziak, P., Mahone, C. R., Löwe, J. \& Goley, E. D. FzlA, an essential regulator of FtsZ filament curvature, controls constriction rate during Caulobacter division. Mol. Microbiol. 107, 180-197 (2018).

3. Thanbichler, M., Iniesta, A. A. \& Shapiro, L. A comprehensive set of plasmids for vanillate- and xylose-inducible gene expression in Caulobacter crescentus. Nucleic Acids Res. 35, e137 (2007).

4. Thanbichler, M. \& Shapiro, L. MipZ, a Spatial Regulator Coordinating Chromosome Segregation with Cell Division in Caulobacter. Cell 126, 147-162 (2006).

5. Figueroa-Cuilan, W., Daniel, J. J., Howell, M., Sulaiman, A. \& Brown, P. J. B. MiniTn7 Insertion in an Artificial attTn7 Site Enables Depletion of the Essential Master Regulator CtrA in the Phytopathogen Agrobacterium tumefaciens. Appl. Environ. Microbiol. 82, 5015-5025 (2016).

6. Choi, K.-H. et al. Genetic tools for select-agent-compliant manipulation of Burkholderia pseudomallei. Appl. Environ. Microbiol. 74, 1064-1075 (2008).

7. Goley, E. D. et al. Assembly of the Caulobacter cell division machine. Mol. Microbiol. 80, 1680-1698 (2011).

8. Meier, E. L. et al. FtsEX-mediated regulation of the final stages of cell division reveals morphogenetic plasticity in Caulobacter crescentus. PLOS Genet. 13, e1006999 (2017) 
188 9. Howell, M. L. et al. Agrobacterium tumefaciens divisome proteins regulate the

189 transition from polar growth to cell division. bioRxiv 412759 (2018).

190 doi: $10.1101 / 412759$

191 10. Watson, B., Currier, T. C., Gordon, M. P., Chilton, M. D. \& Nester, E. W. Plasmid

192 required for virulence of Agrobacterium tumefaciens. J. Bacteriol. 123, 255-264

193 (1975).

194 11. Evinger, M. \& Agabian, N. Envelope-associated nucleoid from Caulobacter

195 crescentus stalked and swarmer cells. J. Bacteriol. 132, 294-301 (1977).

196 12. Modell, J. W., Kambara, T. K., Perchuk, B. S. \& Laub, M. T. A DNA Damage-

197 Induced, SOS-Independent Checkpoint Regulates Cell Division in Caulobacter

198 crescentus. PLOS Biol. 12, e1001977 (2014). 
A

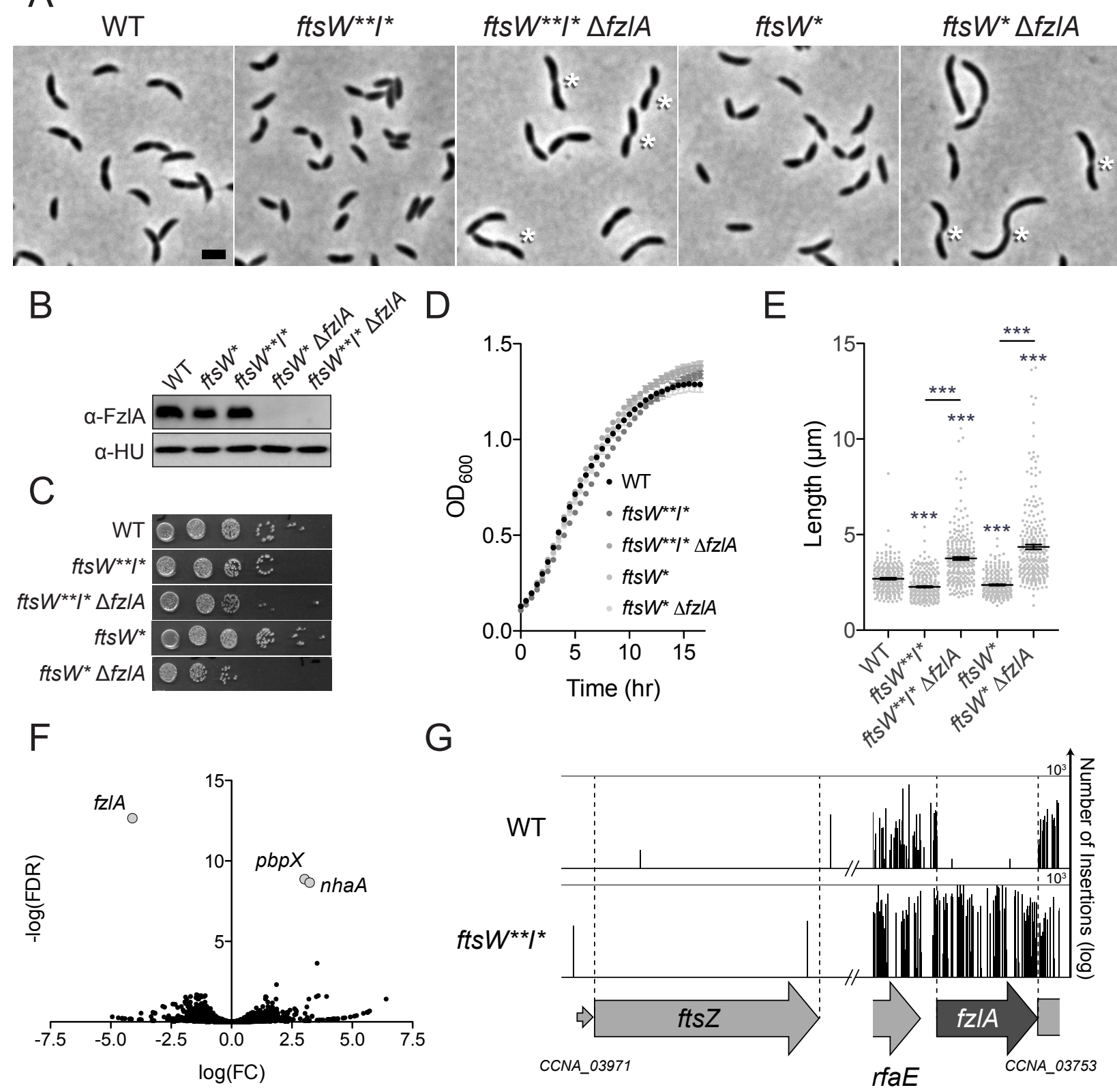

Fig. 1: Hyperactive ftsWI mutants suppress loss of $f z l A$ 

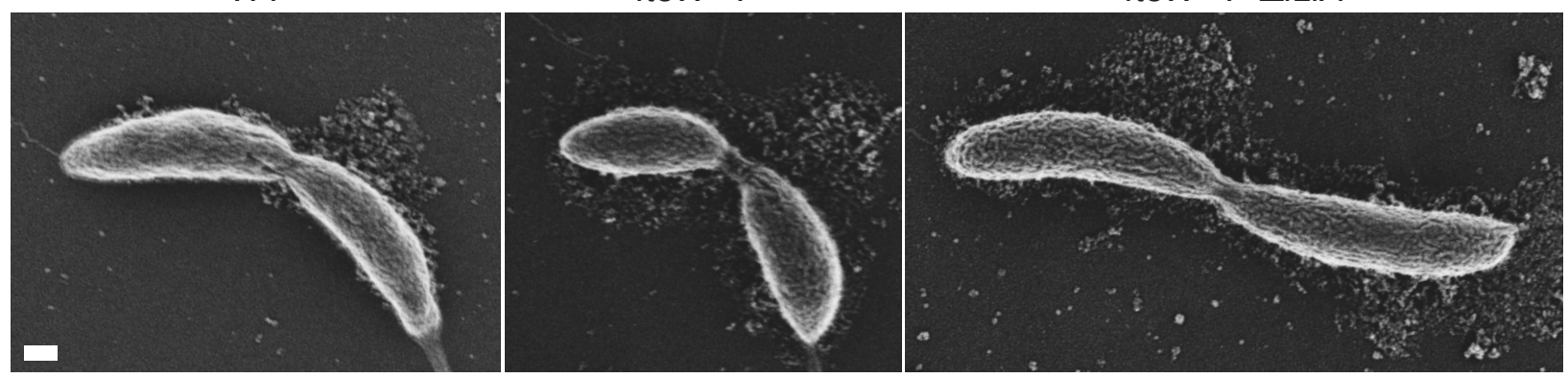

B

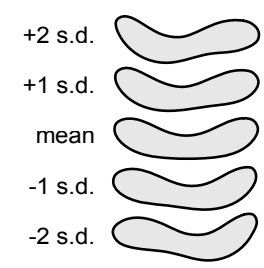

Shape Mode 3 ( $1.9 \%$ of total variance)

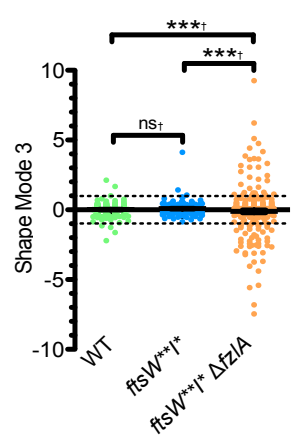

C

D
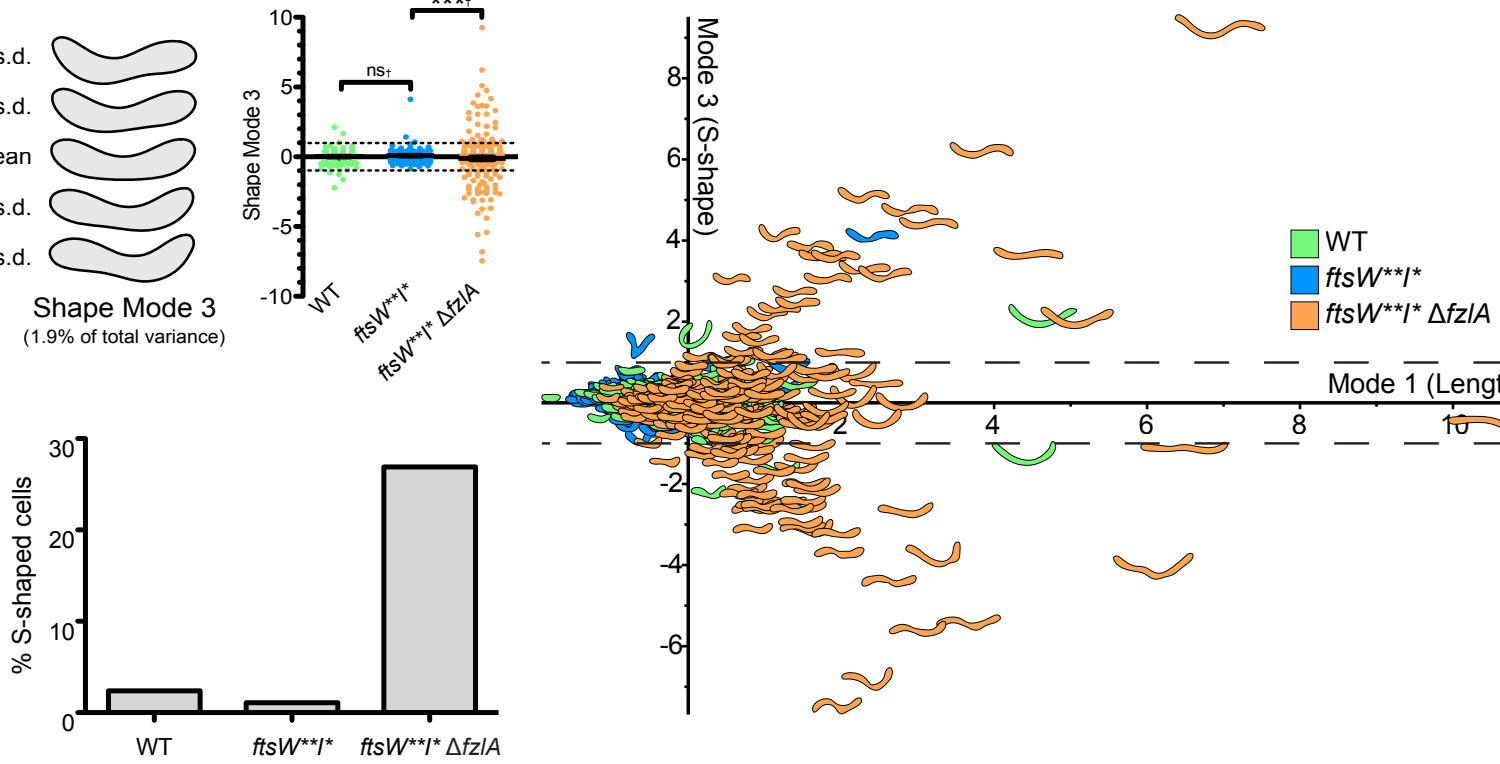

$E$

$\left.f t s W^{* *}\right|^{*} \Delta f z \mid A$
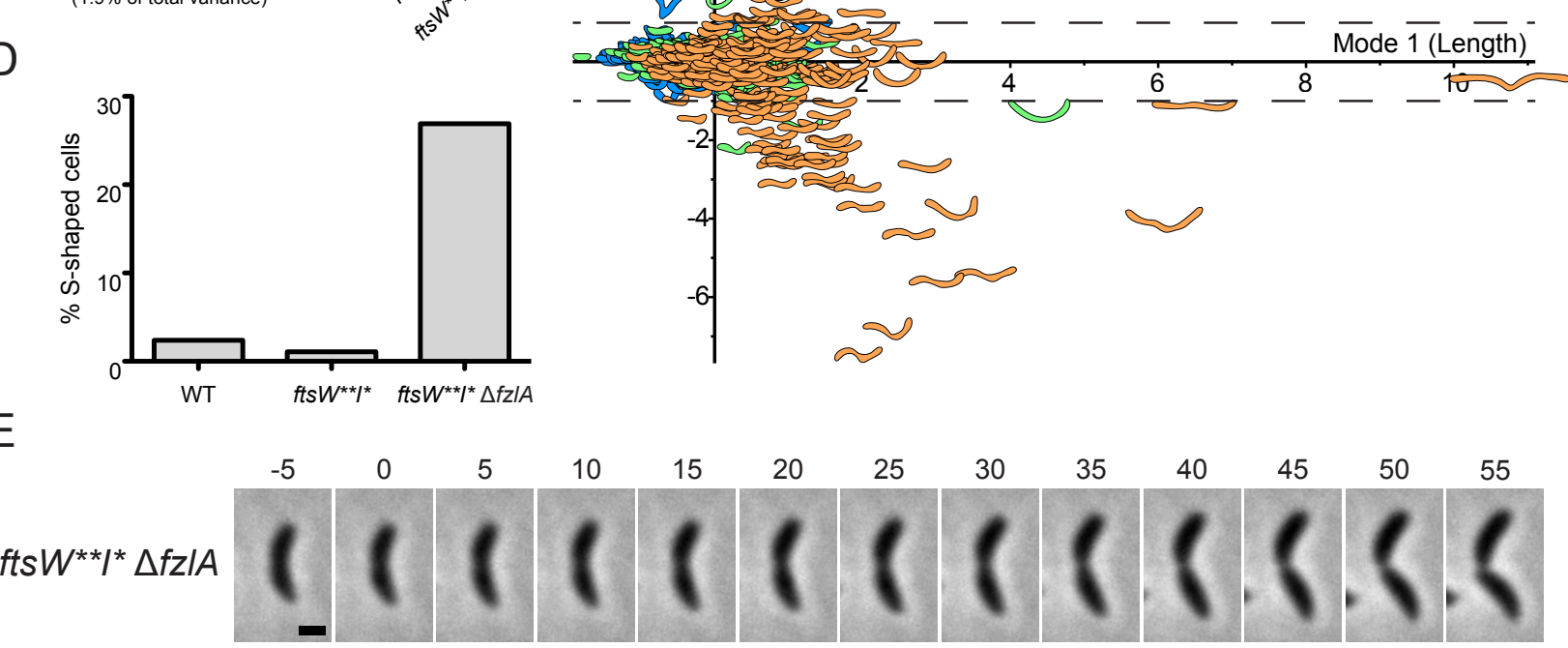

Fig. 3: $f z \mid A$ is required for global shape maintenance 
A + Cephalexin

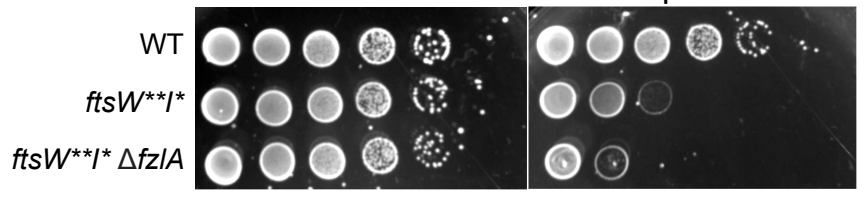

B

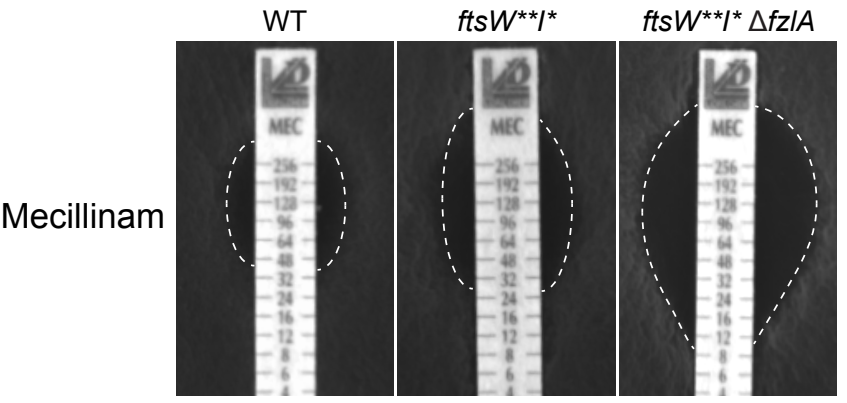

Fig. 4: Loss of $f z / A$ leads to increased cell wall antibiotic sensitivity 
A
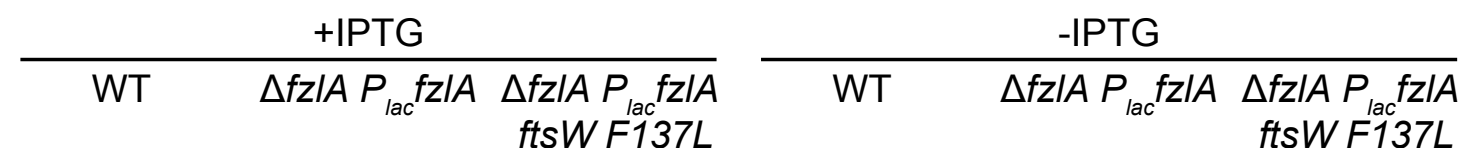
ftsW F137L

$16 \mathrm{hr}$
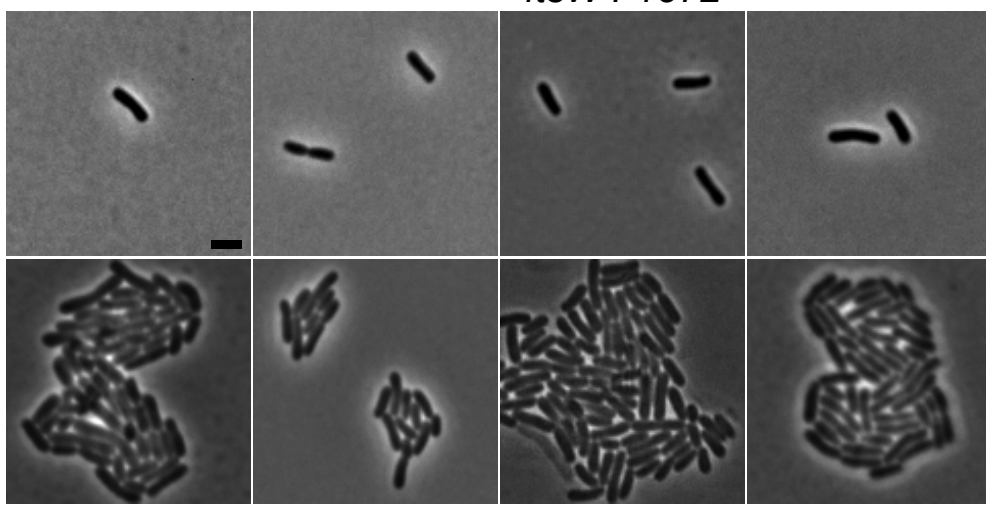
ftsW F137L

B

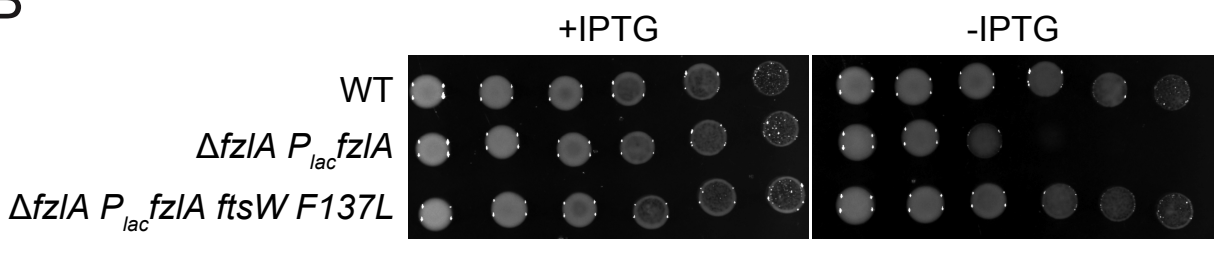

Fig. 5: The ability of hyperactive $f t s W$ to suppress loss of $f z \mid A$ is conserved 


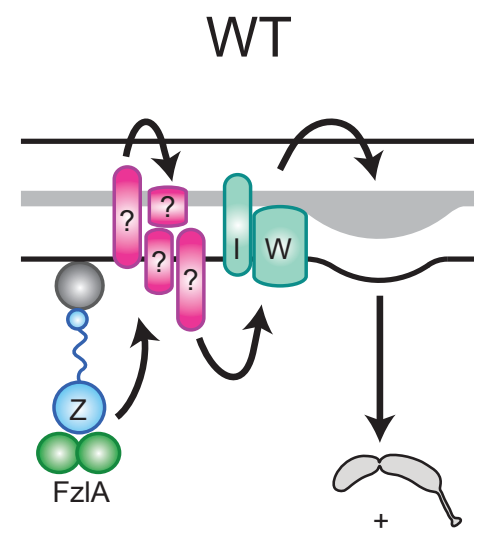

Normal

Constriction

$\beta$-lactam

resistance $f t s W^{* *} I^{*}$

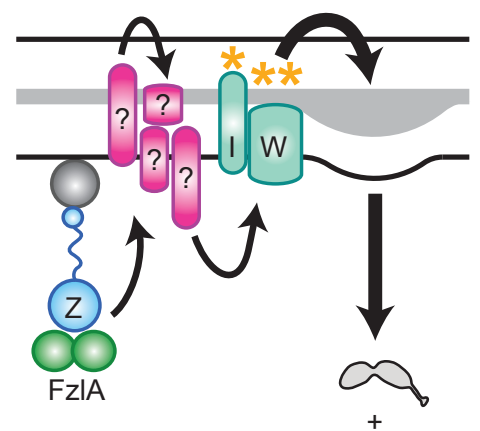

Fast

Constriction<smiles>[AlH2]</smiles>

$\beta$-lactam

sensitivity $f t s W^{* *} I^{*} \Delta f z \mid A$

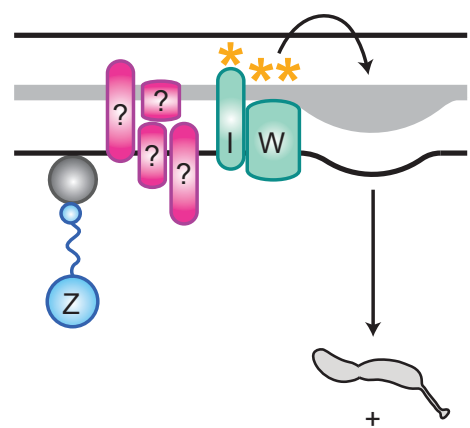

Slow

Constriction

Increased $\stackrel{+}{\beta}$-lactam sensitivity

Fig. 6: FzIA is required for activation of FtsWI and regulates the geometry of PG insertion 
A Blot from Figure 1

B Blot from Figure S2

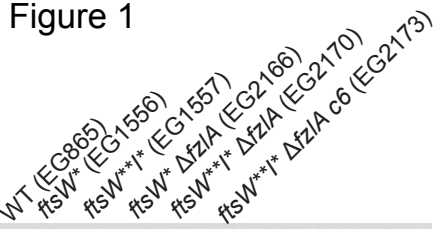

$\alpha-F z \mid A$

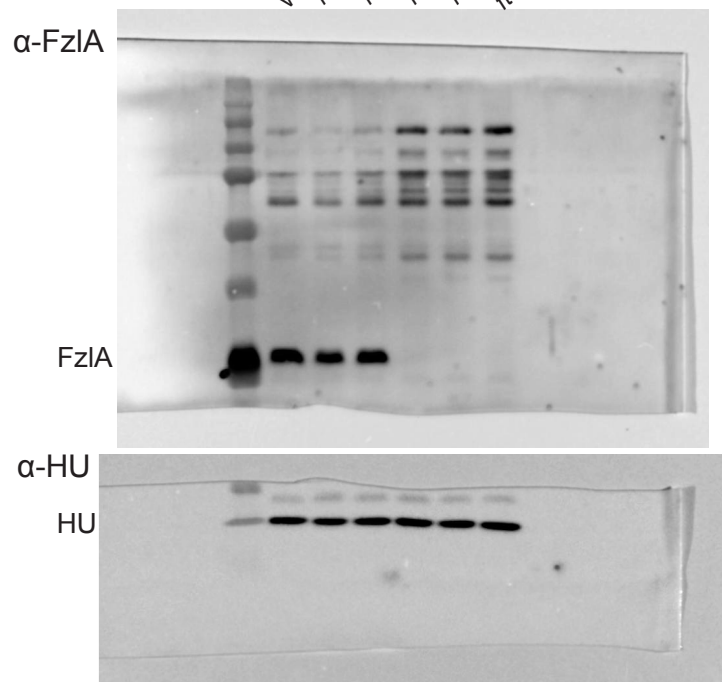

$\alpha-F z \mid A$
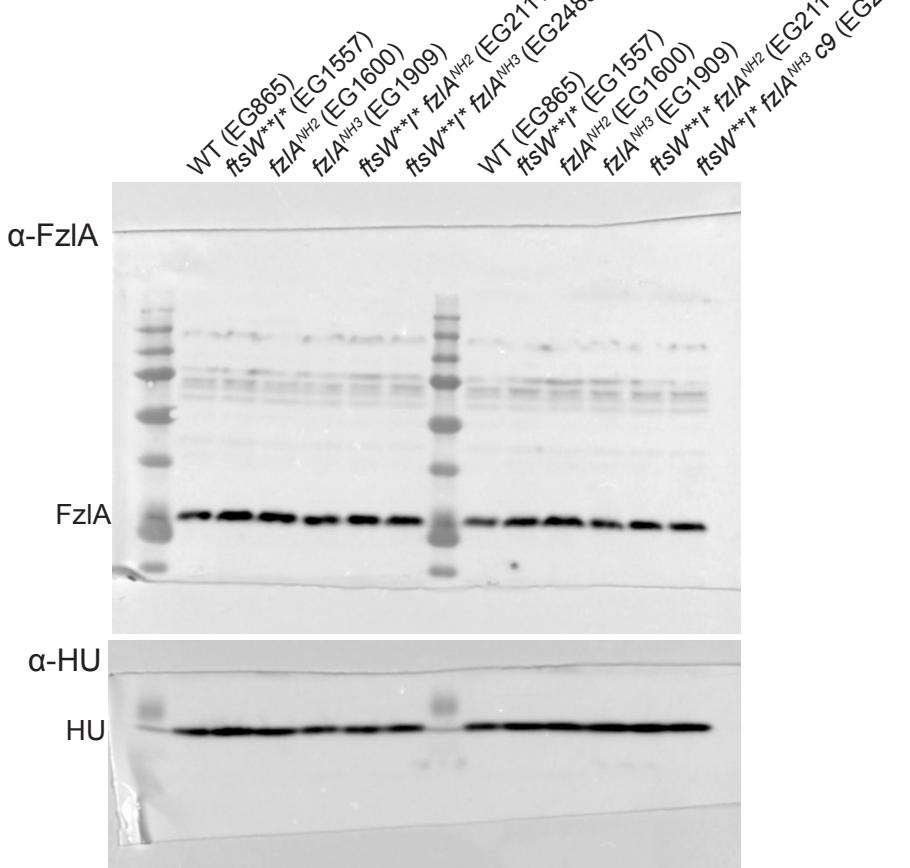

C

Blot from Figure S3

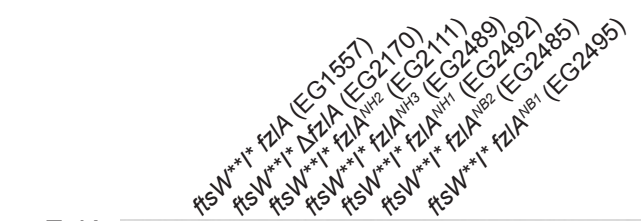

$\alpha-F z \mid A$

FzIA
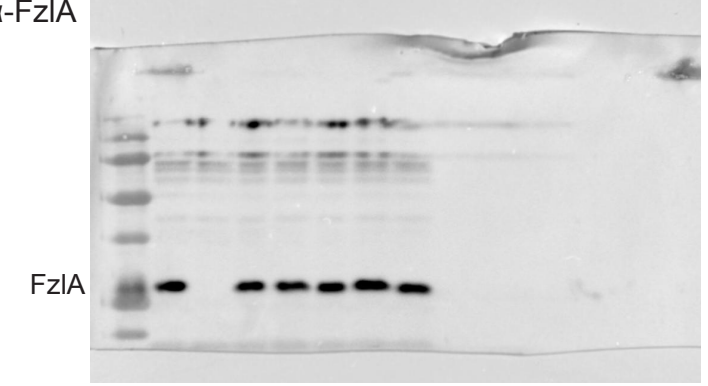

$$
\begin{gathered}
\alpha-H U \\
H U
\end{gathered}
$$

Fig. S1: Uncropped Immunoblots 
$f t s W^{* *} l^{*} \quad f t s W^{* *} l^{*} \quad \mathrm{~B}$
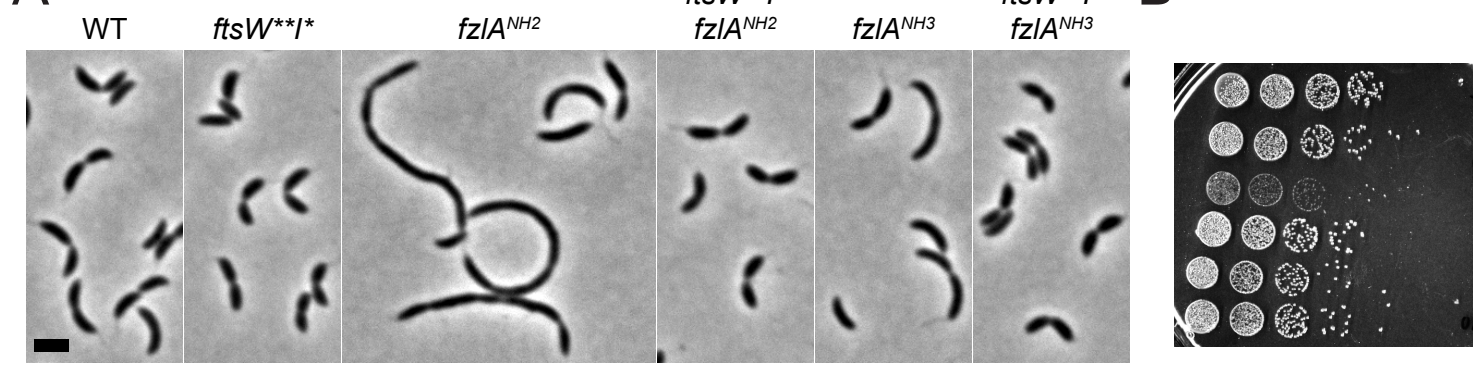

WT

fts $W^{* \star} I^{*}$

$f Z \mid A^{N H 2}$

$f Z \mid A^{N H 3}$

$f t s W^{* *} I^{*} f z \mid A^{N H 2}$

$f t s W^{\star *} I^{*} f z \mid A^{N H 3}$

C

0

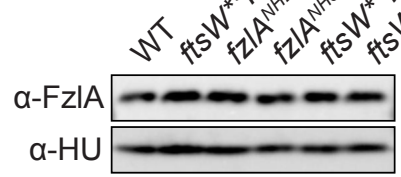

E

- WT

- $f t W^{\star *} I^{\star} f z I A^{N H 2}$

- $f t W^{\star *} I^{*} f z I A^{N H 3}$

- $f t s W^{\star \star} I^{\star}$

- $f z I A^{N H 3}$

- $f z \mid A^{N H 2}$

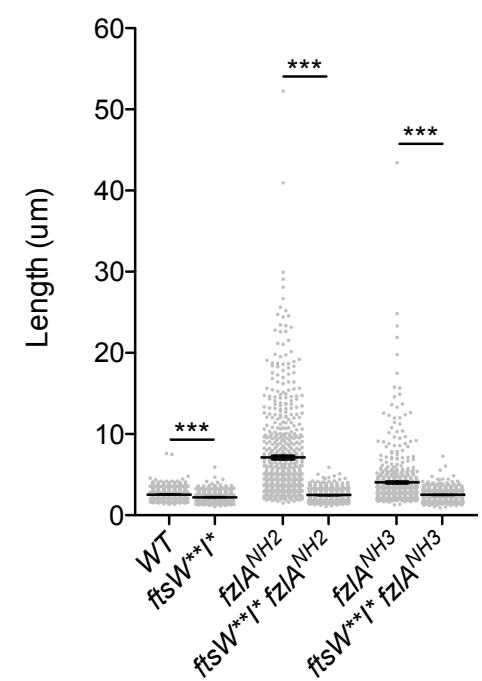

F

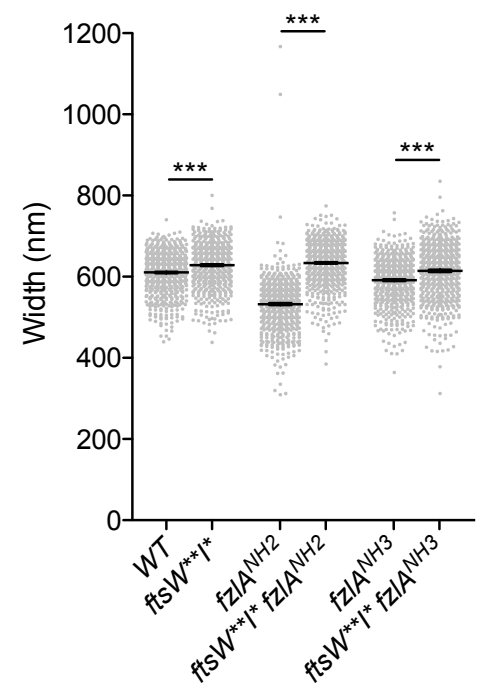

Fig. S2: $\left.f t s W^{* *}\right|^{\star}$ rescue the fitness/morphological defects of two $f z / A$ mutants 


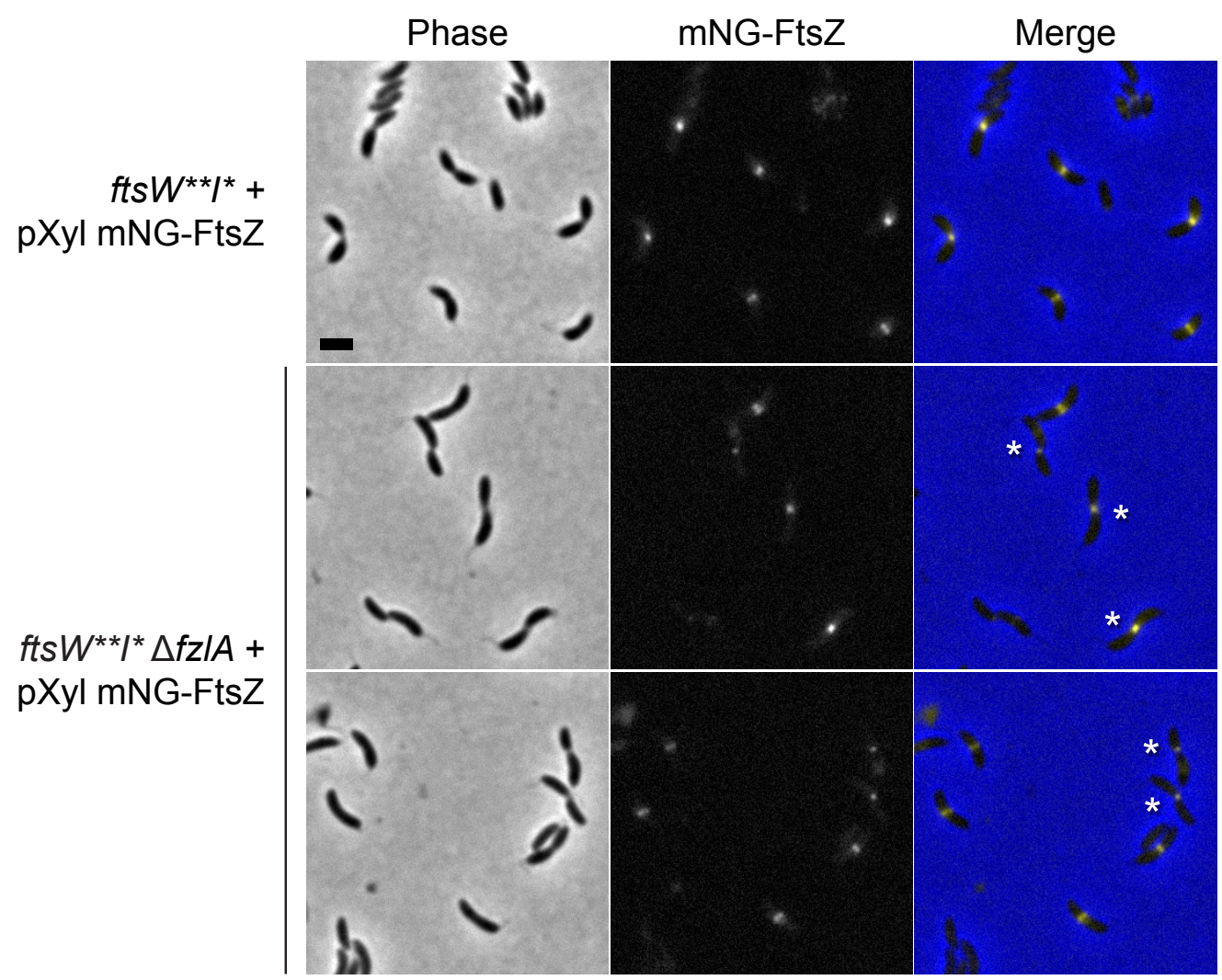

Fig. S4: FtsZ localization is unaffected in $\left.f t s W^{* *}\right|^{*} \Delta f z \mid A$ cells 


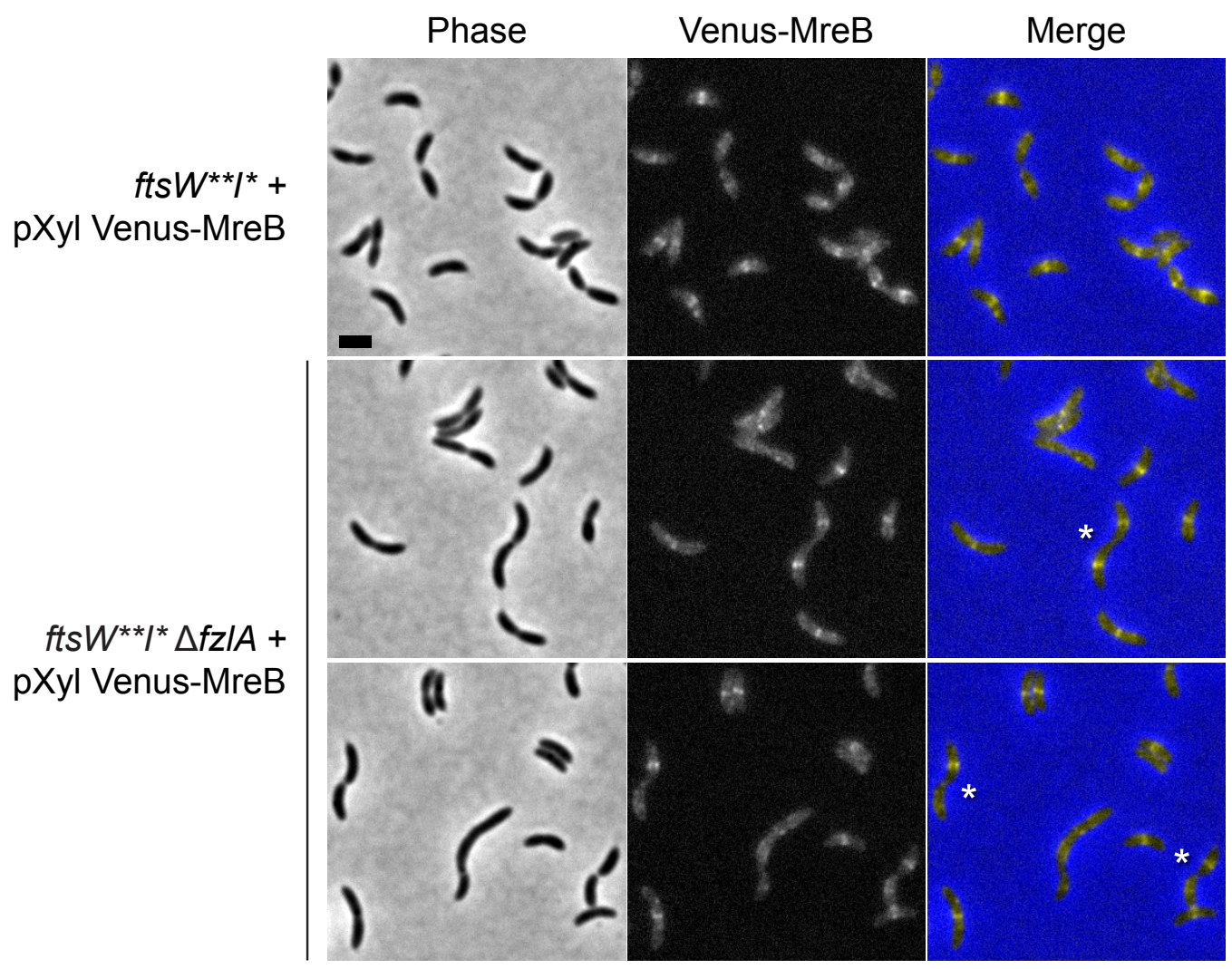

Fig. S5: MreB localization is unaffected in $f t s W^{\star \star} I^{\star} \Delta f z \mid A$ cells 


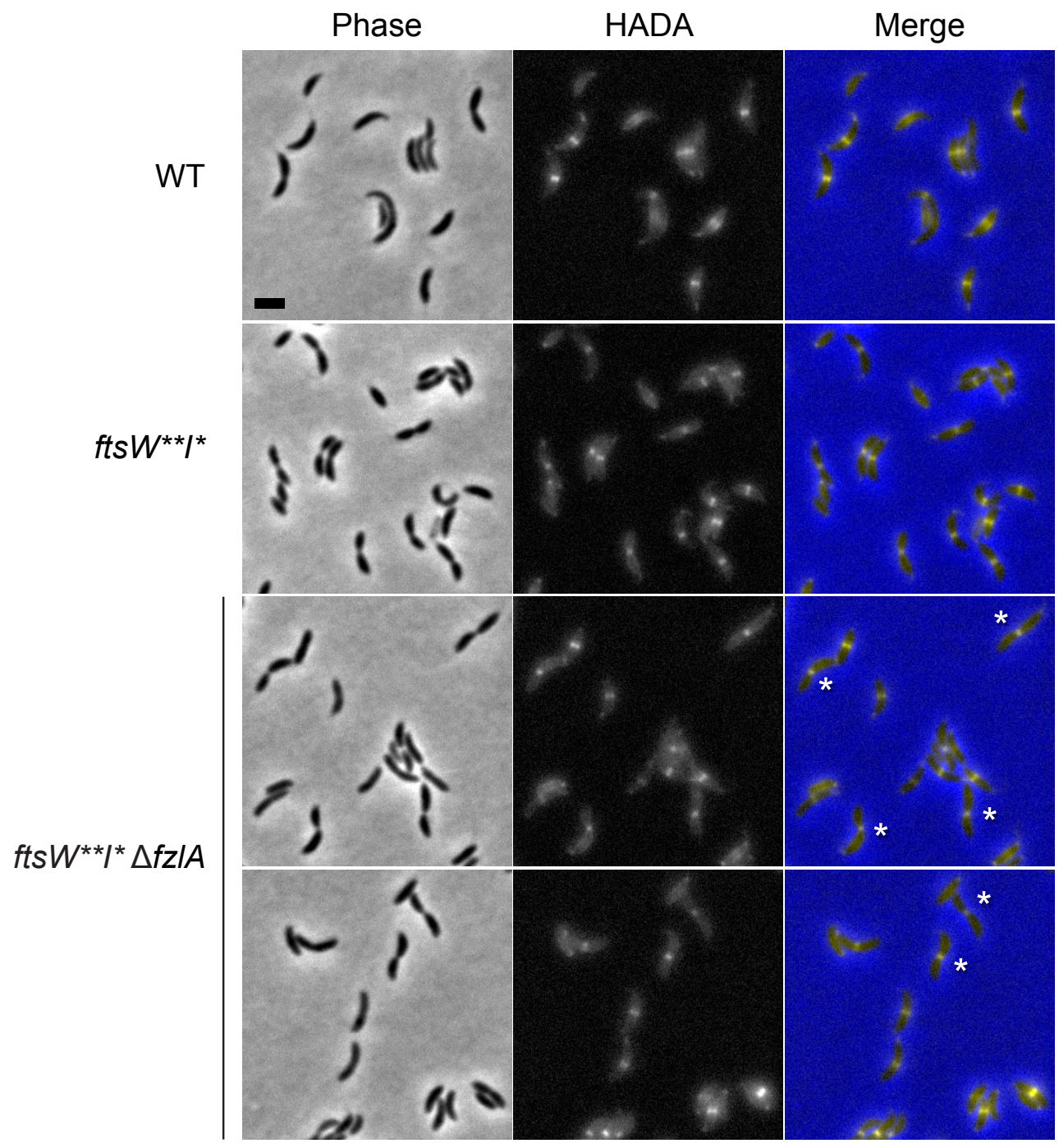

Fig. S6: The localization of $P G$ synthesis is unaffected in $\left.f t s W^{* *}\right|^{*} \Delta f z \mid A$ cells 
A

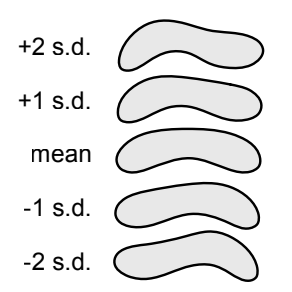

Shape Mode 3

(1.4\% of total variance)

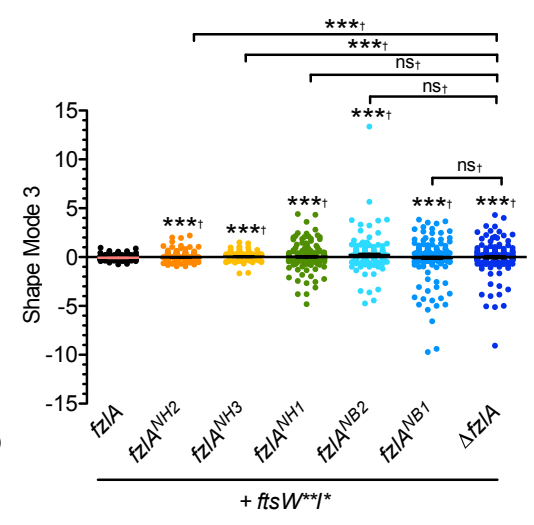

B
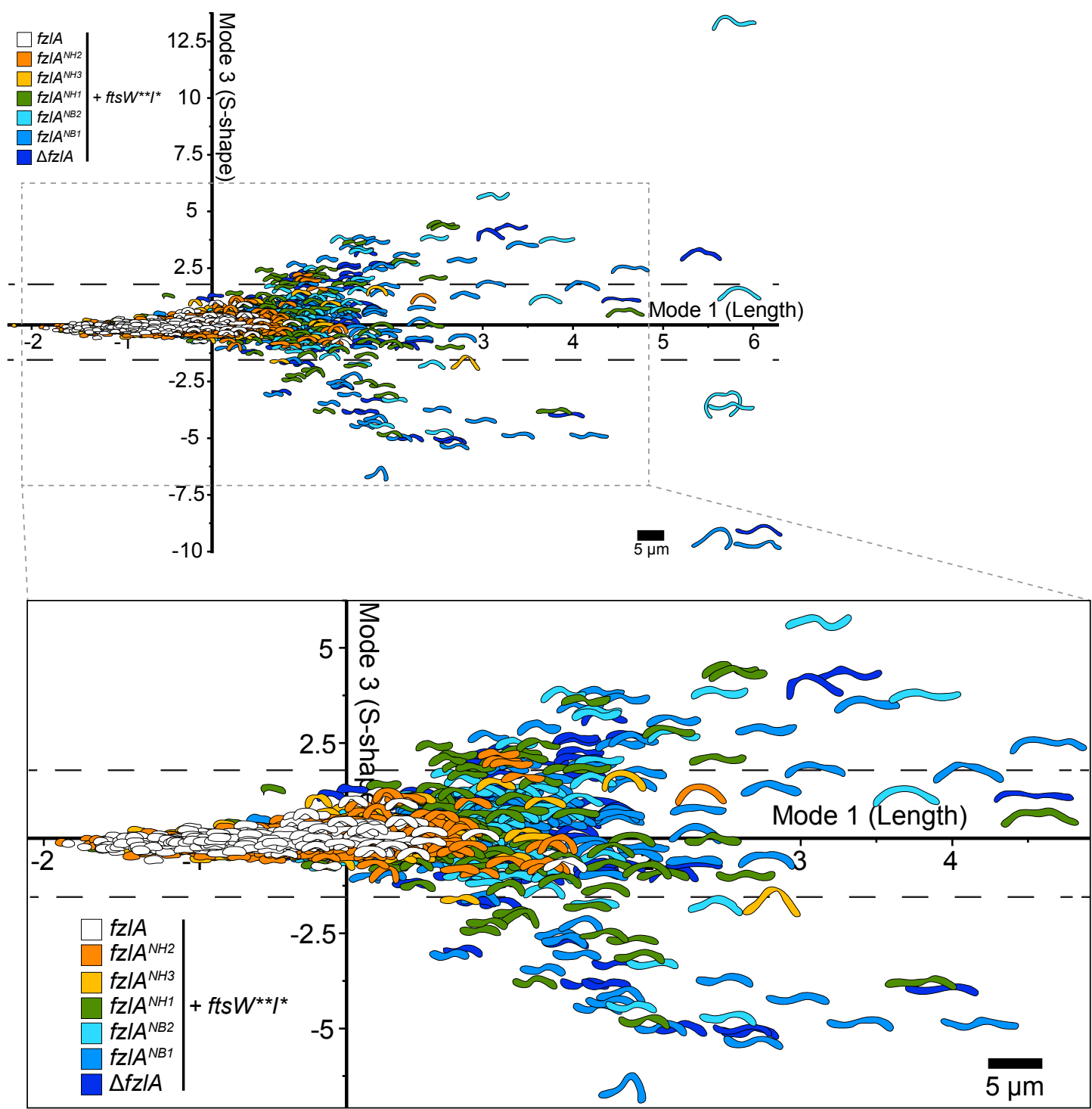

Fig. S7: Interaction of FtsZ with FZIA is necessary for proper division site shape maintenance 


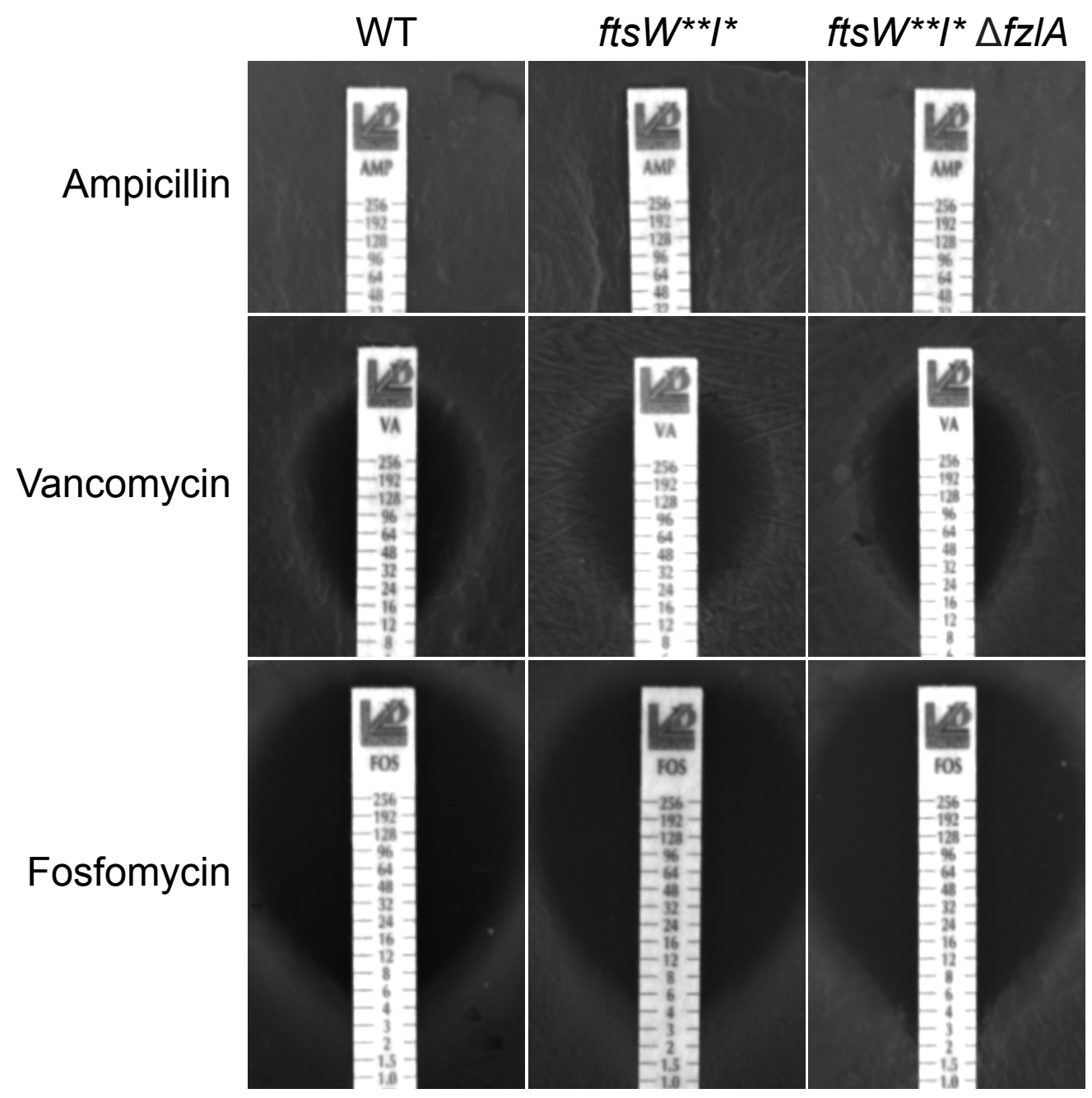

Fig. S8: Loss of $f z I A$ does not confer increased sensitivity to various classes of antibiotics 


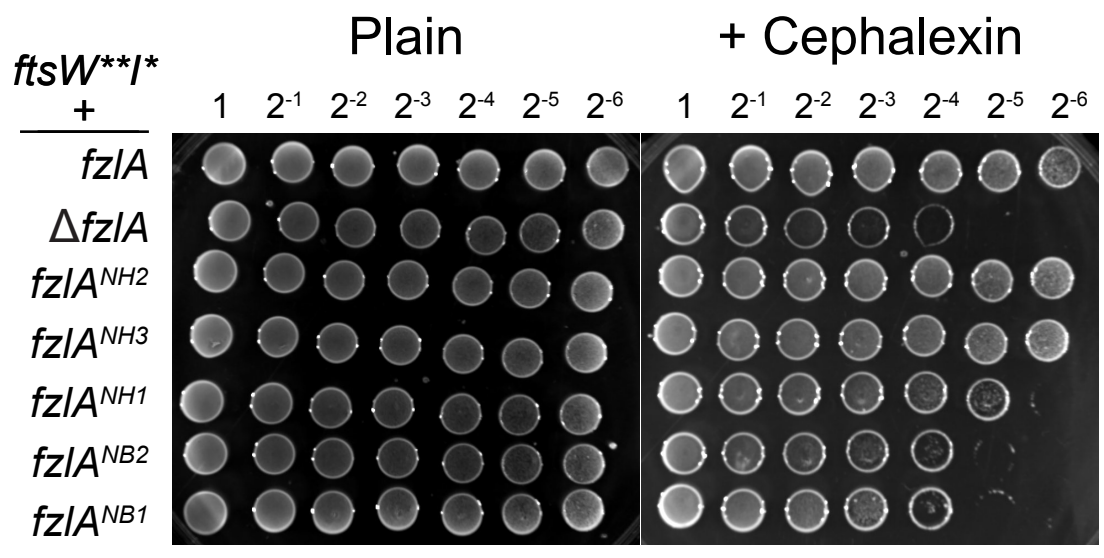

Fig. S9: Interaction of FtsZ with FzIA is necessary for increased resistance to cell wall antibiotics 
A

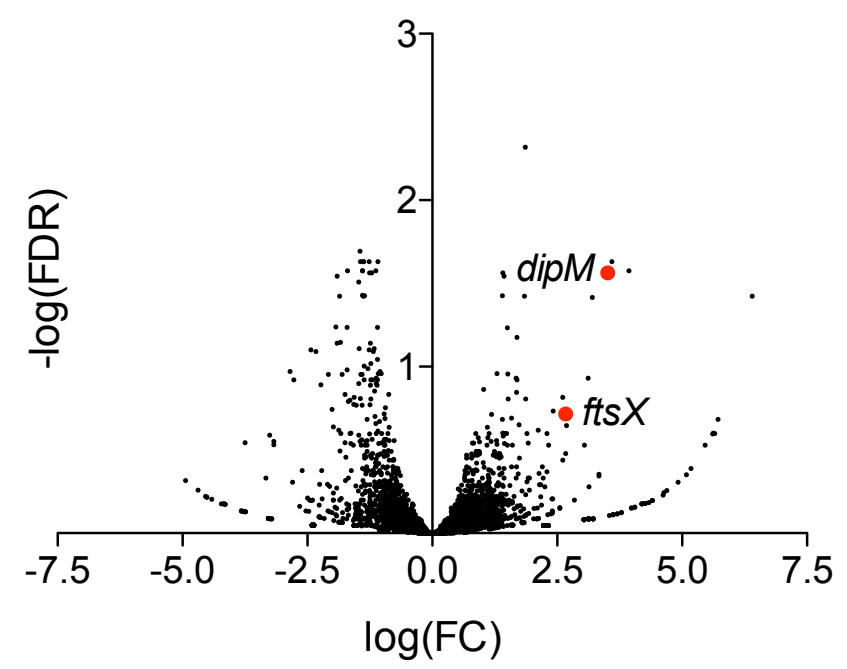

B

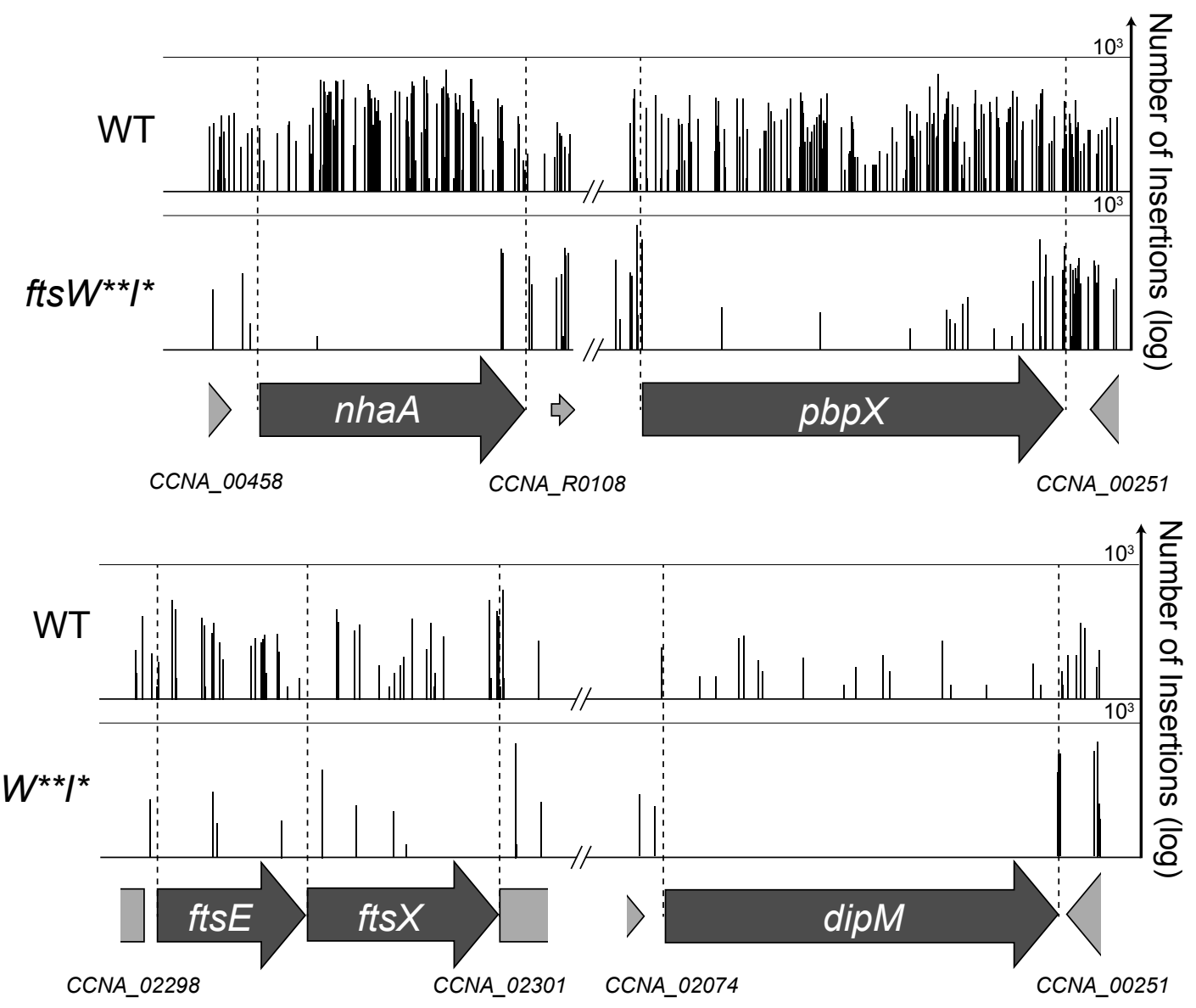

Fig. S10: Multiple non-essential division genes become essential in a hyperactive PG synthase background 
